\title{
Consistent values and the core in continuum market games with two types of players
}

\author{
Sigal Leviatan* \\ 15 Shmuel Ha’Nagid st., Herzelia 46498, ISRAEL (email: sigall@consumer.org.il) \\ Received: October 1998
}

\begin{abstract}
The consistent value is an extension of the Shapley value to the class of games with non-transferable utility.

In this paper, the consistent value will be characterized for market games with a continuum of players of two types. We will show that for such games the consistent value need not belong to the core, and provide conditions under which there is equivalence between the two concepts.
\end{abstract}

Key words: cooprative games, consistent value, continuum games, equivalence theorem, NTU-value

\section{Introduction}

Over the years, many types of values and solutions have been introduced for the class of market games. Some have been shown to be equivalent, under certain conditions, like the "Core Equivalence Theorem" and the "Value Equivalence Theorem" for the Shapley NTU-value (see [1], [11]). Others have been shown to differ (see, for example, [5], showing that the Harsanyi value and the core need not coincide).

In this paper, we characterize Maschler and Owen's consistent value for market games with a continuum of players. The main concept defining the

\footnotetext{
* This thesis was completed under the supervision of Professor Sergiu Hart, The Center for Rationality and Interactive Decision Theory, Department of Mathematics, Department of Economics, The Hebrew University of Jerusalem. I would like to thank Professor Hart for introducing me to this area of research, for his help and guidance, and, especially, for all his patience.

I would also like to thank Michael Borns for improving the style, and an anonymous referee for helpful comments.
} 
consistent value, which is an extension of the Shapley value to the class of games with non-transferable utility, ${ }^{1}$ is that of balanced contributions: every player's average ${ }^{2}$ contribution to the other players equals his average gain from the other players.

Next, we examine the relation between the consistent value and the core for market games with a continuum of players of two types. We will see that even for this class of games there is no equivalence between the consistent value and the core. In fact, we will exhibit an abundance of consistent values which will all reside in the ConvCore, ${ }^{3}$ but usually not in the core itself.

On the other hand, we will observe that the limit of the consistent value 4 does belong to the core. It is worth mentioning that comparing the limit of the consistent value ${ }^{5}$ to the core might in fact be the correct approach to establishing an equivalence theorem between the two. This paper does not take the asymptotic approach, but it is reasonable to conjecture that such an approach might lead to precisely this limit.

Section 2 gives the basic model, the notation, and the assumptions (with standard notation stated explicitly in Appendix A). Section 3 is devoted to definitions, specific notations, and preliminary results (special attention should be given to the definition of the consistent value in Section 3.2, and to the basic results for games with a continuum of players of two types, shown in Section 3.3). The main theorems of this paper are stated (and partially proved) in Section 4. The special case of a unique core point is discussed in Section 5. In Section 6 we examine the flow of the consistent value, and prove the main theorems of this paper. Section 7 is devoted to a number of examples.

\section{The model}

An NTU (non-transferable utility) game form ${ }^{6}$ with a continuum of players of $n$ types is a point-to-set map $V: R_{+}^{n} \rightarrow P\left(R^{n}\right)$, i.e., $V(x) \subset R^{n}$ for every $x \in R_{+}^{n}$. The interpretation of this setup is as follows:

- Every $x \in R_{+}^{n}$ stands for the vector of total masses of each of the $n$ types, and is called a profile or a coalition ${ }^{7}$ (we have a continuum of players of each type).

- A point $a \in V(x)$ represents a vector of per capita utility payoffs that a coalition of profile $x$ can attain (all players of the same type are treated equally); that is, each player of type $i$ (there are $x^{i}$ such players) gets a payoff $a^{i}$.

\footnotetext{
1 This extension is defined in detail later in the paper.

${ }^{2}$ In an NTU game this will be a weighted average, with the weights themselves defined as part of the consistent value.

${ }^{3}$ The term ConvCore is defined precisely later in the paper. In short, it refers to all efficient payoff vectors that either belong to the convex hull of the core or Pareto dominate some point in it.

${ }^{4}$ The exact sense of limit is defined later in the paper. In general, for every proportion between the masses of all types of players we will examine the limit of the consistent value as the total mass of players goes to infinity or to zero.

${ }^{5}$ It is important to realize that the limit of the consistent values is in itself not necessarily a consistent value, as will be seen in Section 6.2.

${ }^{6}$ A game form differs from a game in not specifying the set of players, i.e., the grand coalition.

7 These two terms will be used interchangeably.
} 
- For every type $i$, we will define $r_{i}:=\max \left(V^{i}\left(e^{i}\right)\right),{ }^{8}$ i.e., the per capita utility payoff the players of type $i$ can attain on their own, ${ }^{9}$ and we will assume $r_{i}>0 .{ }^{10} r_{i}$ will be called the individually rational level, and will denote the minimal utility payoff a player of type $i$ is willing to accept.

We denote this game form $[n ; V]$.

Throughout this paper we will assume the following basic assumptions on the function $V$ (and functions derived therefrom). The reader may refer to Hart-Mas-Colell ([3], [4]) for further discussion of these assumptions, and to Appendix A for standard notations which are not explicitly mentioned in the text.

Denote $\hat{V}(x):=\left\{b \in R^{n} \mid b=a * x\right.$ for some $\left.a \in V(x)\right\}$, the set of attainable vectors of total payoffs to each type, and $\hat{V}_{0}(x):=\hat{V}(x) \cap R_{+}^{n}$. Define $v(x, q):=\sup \{q \cdot a \mid a \in V(x)\}$, the support function of the set $V(x)$, and equivalently $\hat{v}(x, q)$ and $\hat{v}_{0}(x, q)$ for $\hat{V}(x)$ and $\hat{V}_{0}(x)$.

Denote also $\hat{D}^{+}:=\left\{(x, q) \in R_{++}^{n} \times R_{+}^{n} \mid \hat{v}(x, q)=q \cdot b\right.$ for some $\left.b \in \hat{V}_{0}(x)\right\}$, the points of the effective domain of $\hat{v}$ where $\hat{v}(x, q)$ is reached at some nonnegative vector.

1. For every $x \in R_{+}^{n}$, the set $V(x)$ is closed, convex and comprehensive. ${ }^{11}$ Moreover, $V(x) \neq \varnothing, R^{n}$. Also, if $x^{i}=0$ and $a \in V(x)$, then $b \in V(x)$ for any $b$ with $b^{j}=a^{j}$ for all $j \neq i$.

2. $\hat{V}_{0}(x)+\hat{V}_{0}\left(x^{\prime}\right) \subset \hat{V}_{0}\left(x+x^{\prime}\right)$, for all $x, x^{\prime} \in R_{+}^{n}$. Notice that this implies that the payoff vector $r=\left(r_{1}, r_{2}, \ldots, r_{n}\right)$ is always feasible, i.e., $r \in V(x)$, for all $x \in R_{++}^{n}$.

3. For every $x \in R_{++}^{n}$ there is a compact set ${ }^{12} C \subset \Delta_{++}^{n}$ such that: ${ }^{13}$

$$
\left\{q \in \Delta_{++}^{n} \mid \hat{v}(x, q)=q \cdot b \text { for some } b \in \hat{V}_{0}(x)\right\} \subset C .
$$

4. For every $x \in R_{++}^{n}$ the support function $\hat{v}(x, \cdot)$ is strictly sub-additive, i.e., $\hat{v}(x, q)+\hat{v}\left(x, q^{\prime}\right)>\hat{v}\left(x, q+q^{\prime}\right)$ whenever $q$ and $q^{\prime}$ are not collinear.

5. The gradient $\nabla_{x} \hat{v}_{0}(x, q)$ exists for every $(x, q) \in \hat{D}^{+}$.

6. The domain $\hat{D}^{+}$has a non-empty interior and the function $\hat{v}(x, q)$ is $C^{2}$ on $\hat{D}^{+}$. Moreover, $\nabla_{q q} \hat{v}(x, q)$ has full possible rank $n-1$, and its minimal nonzero eigenvalue is positive and bounded away from zero in any bounded subset of $\hat{D}^{+}$that is bounded away from the origin. I.e., on any set of the form $\hat{D}^{+} \cap\left(\left\{x \in R_{++}^{n}: \beta \leq\|x\| \leq \gamma\right\} \times \Delta_{+}^{n}\right)$ for some $0<\beta \leq \gamma<\infty$.

7. $V(t x)=V(x)$, for every $x \in R_{+}^{n}$, and $t>0$.

Most of these assumptions are natural to the economic example, where each type $i$ has a utility function $u_{i}$, and an initial endowment, and the every

\footnotetext{
${ }^{8} e^{i}$ is the $i^{\prime}$ th unit vector, and $V^{i}\left(e^{i}\right)$ is the $i^{\prime}$ th component of the utility vector the coalition $e^{i}$ can attain.

${ }^{9}$ For the case of market games, this is the utility of the initial endowment of that player.

${ }^{10}$ Having $r_{i}>0$ does not add any assumptions, because our theorem is invariant to changes of the form $V^{\prime}(x)=V(x)-\left\{a_{0}\right\}$ (keep in mind, though, that the way of formulating the assumptions is not invariant, and depends on $\left.r_{i}>0\right)$.

11 That is, $V(x)-R_{+}^{n} \subset V(x)$.

$12 \Delta_{++}^{n}:=\left\{x \in R_{++}^{n} \mid \sum_{i=1}^{n} x^{i}=1\right\}$ is the interior of $\Delta^{n}$.

13 This assumption is known as the non-levelness assumption. Notice that, in contrast to the equivalent assumption in [3], here the set $C$ is a function of $x$.
} 
$v \in V(x)$ is a utility vector derived from a possible allocations of the total initial endowments. ${ }^{14}$ For example, Assumptions 1 and 2 are satisfied if every $u_{i}$ is continuous and concave. Notice that Assumption 3 simply implies nonlevelness, Assumption 4 implies smoothness of the boundary of $V_{0}(x)$, and Assumption 7 is the common assumption of homogeneity. For a more elaborate discussion of these assumptions and their interpretation see [3, Section II].

Because of the importance of this last assumption, an NTU game form satisfying it will be called an NTU homogeneous game form. The most important class of game forms satisfying this condition are market game forms.

Notice that Assumptions 5 and 6 are satisfied only by strictly convex games, which thus rules out Hyperplane game forms (henceforth, Hyperplane games will be referred to as H-games). The preliminary results, in Section 3, will be proved also for H-games.

Definition 1. A payoff configuration for the game form $[n ; V]$ is a function $a: R_{++}^{n} \rightarrow R_{++}^{n}$ that assigns to every coalition $x \in R_{++}^{n}$ a payoff vector $a(x) \in R_{++}^{n}$, i.e., each player of type $i$ receives a payoff of $a^{i}(x)$.

\section{Preliminaries}

\subsection{Core and ConvCore}

The concept of the core is defined here for the sake of completeness.

Definition 2. Let $[n ; V]$ be an NTU game form with a continuum of players. The core of the game is a point-to-set map that assigns to every coalition $x \in R_{++}^{n} a$ set of attainable payoff vectors, ${ }^{15}$ such that no sub-coalition of $x$ can attain a higher payoff for all its players. That is: ${ }^{16}$

$a \in \operatorname{core}(x, V) \Leftrightarrow \nexists y \leq x, b \in V(y)$ such that $a<b$.

In this paper we will also be using an extension of this concept:

Definition 3. Let $[n ; V]$ be an NTU game form with a continuum of players. Define ConvCore as a point-to-set map that assigns to every coalition $x \in R_{++}^{n}$ a set of attainable payoff vectors, in the following manner. ${ }^{17}$

$$
\operatorname{ConvCore}(x, V):=\left(\operatorname{conv}(\operatorname{core}(x, V))+R_{+}^{n}\right) \cap \partial V(x) ;
$$

that is, the set of all efficient payoffs $a$ (i.e., $a \in \partial V(x))$ greater than or equal to some convex combination of core points. See Figure 1 for an example of this concept.

We add to this another important concept: individual rationality.

\footnotetext{
${ }^{14}$ Allowing endowment to be wasted (i.e. not allocated to any type) is what justifies comprehensiveness.

15 I.e., $\operatorname{core}(x, V) \subset V(x)$.

16 By Assumption 3, if there exists $y \leq x$ and $b \in V(y)$ such that $a<b$, then there exists $c \in V(y)$ such that $a \ll c$.

${ }^{17} \operatorname{Conv}(A)$ means the convex hull of the set $A . \partial V(x)$ means the boundary of $V(x)$.
} 


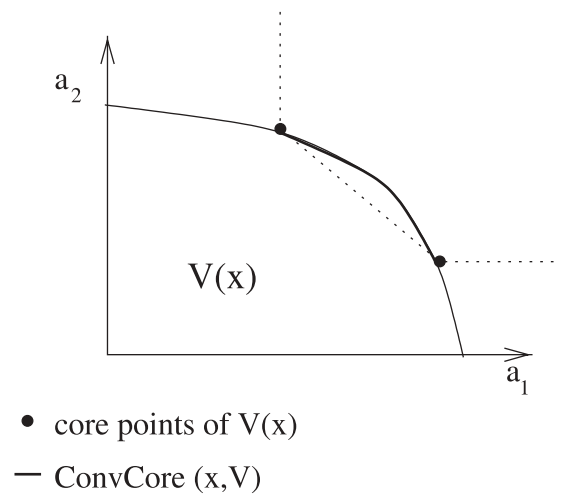

Fig. 1. ConvCore $(x, V)$

Definition 4. A payoff vector $a(x) \in R_{++}^{n}$, for a coalition $x \in R_{++}^{n}$, is individually rational if and only if every player (i.e., a player of every type) receives at least the minimal utility payoff that he is willing to accept. That is, ${ }^{18}$

$$
a^{i}(x) \geq r_{i} \quad \forall i
$$

\subsection{Consistent value}

The consistent value was first introduced by Maschler-Owen (see [8], [9]) as an extension of the Shapley value to the class of $N T U$ games.

A characterization of the consistent value for games with a finite set of players is as follows (see [2, Proposition 4]):

Definition 5. Let $(N, V)$ be an NTU finite game, and $\mathbf{a}=\left(a_{S}\right)_{S \subset N}$ a payoff configuration. Then a is a consistent value payoff configuration of $(N, V)$ if and only if for each $S \subset N$ there exists a vector $\lambda_{S} \in R_{++}^{S}$ such that:

- $a_{S} \in \partial V(S)$

- $\lambda_{S} \cdot a_{S}=\operatorname{Max}\left\{\lambda_{S} \cdot c \mid c \in V(S)\right\}$;

- $\sum_{j \in S \backslash i} \lambda_{S}^{i}\left(a_{S}^{i}-a_{S \backslash j}^{i}\right)=\sum_{j \in S \backslash i} \lambda_{S}^{j}\left(a_{S}^{j}-a_{S \backslash i}^{j}\right)$ for all $i \in S$.

Notice that the first condition states that the payoff vector $a_{S}$ is efficient for the coalition $S$, and the second condition ensures that $\lambda_{S}$ is a support vector of $V(S)$ at the point $a_{S}$. The third condition somewhat resembles the property of balanced contributions (see [10]) of the Shapley value, which requires equality between the weighted sum of the $i$ 'th player's contribution to the payoffs of the other players and their contributions to his payoff.

This definition can easily be generalized to games with a continuum of players, of a finite number of types:

\footnotetext{
18 Recall that for market games this is the payoff he would have received had he not participated in trading.
} 
Definition 6. Let $[n ; V]$ be an NTU game form with a continuum of players, and let $a: R_{++}^{n} \rightarrow R_{++}^{n}$ be a $C^{1}$ payoff configuration. Then $a$ is a smooth consistent value payoff configuration of $[n ; V]$ if and only if there exists $\lambda: R_{++}^{n} \rightarrow R_{++}^{n}$, such that for each vector $x \in R_{++}^{n}$ (where $\left.N:=\{1, \ldots, n\}\right)$ :

- $a(x) \in \partial V(x)$

- $\sum_{i \in N} \lambda^{i}(x) x^{i} a^{i}(x)=\operatorname{Max}\left\{\sum_{i \in N} \lambda(x) x^{i} c^{i} \mid c \in V(x)\right\}$;

- $\sum_{j \in N \backslash i} \lambda^{i}(x) x^{j} \frac{\partial a^{i}}{\partial x^{j}}(x)=\sum_{j \in N \backslash i} \lambda^{j}(x) x^{j} \frac{\partial a^{j}}{\partial x^{i}}(x)$ for all $i \in N$.

Again, the first condition is that of efficiency, and the second ensures that $\lambda$ is the support vector at every point. Notice that in the third condition the sum is over all players of all types (in Definition 5 there was only one player of each type), hence the multiplication by $x^{j} \cdot{ }^{19}$ Notice that because the definition of the consistent value is local, the value of $V$ on the boundary of $R_{++}^{n}$ is irrelevant to the definition of consistent value.

It is worth mentioning that, owing to the assumption of homogeneity, for every consistent value $a(x)$ and every $t>0$ the payoff configuration $b(x)=$ $a(t x)$ is also a consistent value. Thus if there exists one non-homogeneous consistent value, then in fact there exists a continuum of such. One might wonder therefore why we do not restrict our discussion to homogeneous consistent values, but it turns out that these exist only in the unique case where the core allows for a $C^{1}$ selection, ${ }^{20}$ and that there are many interesting games where this is not true, and still non-homogeneous consistent values exist.

Remark: It is sufficient to replace $\frac{\partial a^{i}}{\partial x^{j}}$ by $\lim _{\Delta x^{j} \rightarrow 0^{+}} \frac{a^{i}(x)-a^{i}\left(x-\Delta x^{j}\right)}{\Delta x^{j}}$ (i.e., differentiability from below) in order to generalize the definition of the consistent value. We will refer to a non- $C^{1}$ payoff configuration which satisfies this modified condition as a semi-smooth consistent value. ${ }^{21}$

Note that while the assumption of $C^{1}$ can be weakened in this manner there is no possibility of discarding the condition of differentiability from below, while keeping the essence of the consistent value.

Proposition 1. Let $[n ; V]$ be a homogeneous continuum game form with $n$ types of players which satisfies our assumptions. Let a be a smooth consistent value. Then:

a belongs to the core, i.e., $a(x) \in \operatorname{core}(x, V)$ for every $x \in R_{++}^{n}$ if and only if a is homogeneous of degree 0.

Proof: By Theorem VII.1 and VII.2 of [5], ${ }^{22}$ for every $\bar{x} \in R_{++}^{n}$ the payoff vector $c \in \partial V(\bar{x})$ with support vector ${ }^{23} \lambda \in R_{++}^{n}$ satisfies

$$
c \in \operatorname{core}(\bar{x}, V) \Leftrightarrow \lambda * c=\nabla_{x} \hat{v}(\bar{x}, \lambda) .
$$

\footnotetext{
19 Actually, the contributions of the other players of type $i$ should be added to the left side of that equation, and this player's contribution to the other players of his type to the right side, but these contributions cancel out.

${ }^{20}$ See Corollary 10 on this point. When homogeneous consistent values do exist they belong to the core, as will be shown by Proposition 1.

21 Contrary to a smooth consistent value defines earlier.

22 This is actually the "value equivalence theorem" for the Shapley NTU value under these assumptions (see [1] and [11]).

23 I.e., $\sum_{i=1}^{n} \bar{x}^{i} \lambda^{i} c^{i}$ is maximal for $c \in V(\bar{x})\left(\sum_{i=1}^{n} \bar{x}^{i} \lambda^{i} c^{i}=\hat{v}(\bar{x}, \lambda)\right)$.
} 
$a: R_{++}^{n} \rightarrow R_{++}^{n}$ is a smooth consistent value. Let $\mu: R_{++}^{n} \rightarrow \Delta_{++}^{n}$ be the corresponding support vectors. This function is well defined since assumptions 5 and 6 guaranty the corresponding support vectors are unique (a strictly convex efficient frontier). Then

$$
\hat{v}(x, \mu(x))=\sum_{i=1}^{n} \mu^{i}(x) x^{i} a^{i}(x) .
$$

At the consistent value, $a(\bar{x})$ :

$$
\begin{aligned}
\left.\frac{\partial \hat{v}(x, \lambda)}{\partial x^{i}}\right|_{(\bar{x}, \mu(\bar{x}))} & =24\left(\left.\frac{\partial \hat{v}(x, \lambda)}{\partial x^{i}}\right|_{(\bar{x}, \mu(\bar{x}))}+\left.\sum_{j=1}^{n} \frac{\partial \hat{v}(x, \lambda)}{\partial \lambda^{j}}\right|_{(\bar{x}, \mu(\bar{x}))} \frac{\partial \mu^{j}}{\partial x^{i}}(\bar{x})\right) \\
& -\left.\sum_{j=1}^{n} \frac{\partial \hat{v}(x, \lambda)}{\partial \lambda^{j}}\right|_{(\bar{x}, \mu(\bar{x}))} \frac{\partial \mu^{j}}{\partial x^{i}}(\bar{x}) \\
= & \left.\frac{d \hat{v}(x, \mu(x))}{d x^{i}}\right|_{\bar{x}}-\left.\sum_{j=1}^{n} \frac{\partial \hat{v}(x, \lambda)}{\partial \lambda^{j}}\right|_{(\bar{x}, \mu(\bar{x}))} \frac{\partial \mu^{j}}{\partial x^{i}}(\bar{x}) \\
= & \left.\frac{d\left(\sum_{j=1}^{n} \mu^{j}(x) x^{j} a^{j}(x)\right)}{d x^{i}}\right|_{\bar{x}}-\sum_{j=1}^{n} \bar{x}^{j} a^{j}(\bar{x}) \frac{\partial \mu^{j}}{\partial x^{i}}(\bar{x}) \\
= & \sum_{j=1}^{n} \mu^{j}(\bar{x}) \bar{x}^{j} \frac{\partial a^{j}}{\partial x^{i}}(\bar{x})+\mu^{i}(\bar{x}) a^{i}(\bar{x}) \\
= & { }^{26} \sum_{j=1}^{n} \mu^{i}(\bar{x}) \bar{x}^{j} \frac{\partial a^{i}}{\partial x^{j}}(\bar{x})+\mu^{i}(\bar{x}) a^{i}(\bar{x})
\end{aligned}
$$

Therefore the consistent value belongs to the core if and only if $\sum_{j=1}^{n} \bar{x}^{j} \frac{\partial a^{i}}{\partial x^{j}}(\bar{x})=0$ for $i=1, \ldots, n$; that is, $a$ is homogeneous of degree 0 .

This proposition is also true for $\mathrm{H}$-games:

Proposition 1a. Let $[n ; V]$ be a continuum market H-game form with $n$ types of players which satisfies Assumptions 1-4 and 7. Let a be a smooth consistent value. Then:

a belongs to the core, i.e., $a(x) \in \operatorname{core}(x, V)$ for every $x \in R_{++}^{n}$ if and only if $a$ is homogeneous of degree 0 .

\footnotetext{
${ }^{24}$ The function $\mu(x)$ is indeed differentiable, since $a$ is $C^{1}$ and $\mu$ is precisely the $C^{1}$ function $p(x, a(x))$ defined in [3, Section VII.2].

${ }^{25}$ See [7, pp. 64-66] and Assumption 6.

${ }^{26}$ By the third condition in the definition of the consistent value.
} 
Proof: $[n ; V]$ is an H-game form, that is,

$$
V(x)=\left\{c \in R_{++}^{n} \mid \sum_{i=1}^{n} \lambda^{i}(x) x^{i} c^{i} \leq g(x)\right\}
$$

for some $g: R_{++}^{n} \rightarrow R_{++}$homogeneous of degree 1 , and $\lambda: \Delta_{++}^{n} \rightarrow R_{++}^{n}$ homogeneous of degree 0 , both continuously differentiable.

Since $a$ is a consistent value, $a(x) \in \partial V(x)$ for every $x \in R_{++}^{n}$. Thus

$$
g(x)=\sum_{j=1}^{n} \lambda^{j}(x) x^{j} a^{j}(x) \quad \forall x \in R_{++}^{n} ;
$$

therefore for every $i \in\{1, \ldots, n\}$ and $\bar{x} \in R_{++}^{n}$

$$
\begin{aligned}
\frac{\partial g}{\partial x^{i}}(\bar{x}) & =\lambda^{i}(\bar{x}) a^{i}(\bar{x})+\sum_{j=1}^{n} \frac{\partial \lambda^{j}}{\partial x^{i}}(\bar{x}) \bar{x}^{j} a^{j}(\bar{x})+\sum_{j=1}^{n} \lambda^{j}(\bar{x}) \bar{x}^{j} \frac{\partial a^{j}}{\partial x^{i}}(\bar{x}) \\
& ={ }^{27} \lambda^{i}(\bar{x}) a^{i}(\bar{x})+\sum_{j=1}^{n} \frac{\partial \lambda^{j}}{\partial x^{i}}(\bar{x}) \bar{x}^{j} a^{j}(\bar{x})+\sum_{j=1}^{n} \lambda^{i}(\bar{x}) \bar{x}^{j} \frac{\partial a^{i}}{\partial x^{j}}(\bar{x}) .
\end{aligned}
$$

The first-order condition for a point $c \in \partial V(\bar{x})$ to be in core $(\bar{x}, V)$ is that for every $i \in\{1, \ldots, n\}$

$$
\left.\frac{\partial\left[\sum_{j=1}^{n} \lambda^{j}(x) x^{j} c^{j}-g(x)\right]}{\partial x^{i}}\right|_{\bar{x}}=0,
$$

that is,

$$
\frac{\partial g}{\partial x^{i}}(\bar{x})=\lambda^{i}(\bar{x}) c^{i}+\sum_{j=1}^{n} \frac{\partial \lambda^{j}}{\partial x^{i}}(\bar{x}) \bar{x}^{j} c^{j} .
$$

Therefore, the consistent value satisfies the first-order conditions of the core if and only if $a$ is homogeneous of degree 0 (i.e., $\sum_{j=1}^{n} \bar{x}^{j} \frac{\partial a^{i}(x)}{\partial x^{j}}(\bar{x})=0$ for every $i \in\{1, \ldots, n\}$ and $\left.\bar{x} \in R_{++}^{n}\right)$.

Under Assumptions 2 and 7 (superadditivity and homogeneity), satisfying the first-order conditions of the core is equivalent to being in the core:

Assume to the contrary that $c \in \partial V(\bar{x})$ satisfies the first-order conditions stated above, and there exist $x \leq \bar{x}\left(x \in R_{++}^{n}\right)$ and $d \in V(x)$ such that ${ }^{28}$ $d=(1+\alpha) c$ for some $\alpha>0$.

27 By the third condition of the consistent value.

${ }^{28}$ If there exists $d_{1} \in V(x)$ such that $c<d_{1}$ then there also exists $d_{2} \in V(x)$ such that $c \ll d_{2}=$ $(1+\alpha) c$. 
Because of homogeneity (Assumption 7), the same is true for $t x$ for every $t>0$; hence we can take $y \in R_{++}^{n}$ such that $\bar{x}+y=t x$ for some $t>0$. So we have:

$$
\begin{aligned}
& c * \bar{x} \in \hat{V}(\bar{x}) \\
& (1+\alpha) c *(\bar{x}+y) \in \hat{V}(\bar{x}+y) ;
\end{aligned}
$$

therefore, for every $\varepsilon>0$ (by superadditivity and homogeneity):

$$
(\bar{x}+\varepsilon y) * c+\varepsilon \alpha(\bar{x}+y) * c \in \hat{V}(\bar{x}+\varepsilon y) .
$$

That is (H-game):

$$
g(\bar{x}+\varepsilon y)-\lambda(\bar{x}+\varepsilon y) \cdot((\bar{x}+\varepsilon y) * c) \geq \varepsilon \alpha \lambda(\bar{x}+\varepsilon y) \cdot((\bar{x}+y) * c) .
$$

Deriving the two sides with respect to $\varepsilon$ (as $\varepsilon$ goes to 0 ), we get 0 on the left side (by the first-order condition of the core) and a positive number $(\alpha \lambda(\bar{x}) \cdot((\bar{x}+y) * c))$ on the right side: a contradiction.

That is, the consistent value belongs to the core if and only if $a$ is homogeneous of degree 0 .

\subsection{Two-type continuum games}

From this point on, the discussion will be restricted to continuum market games with exactly $n=2$ types of players (the case of $n=1$ types is of no interest, for reasons of symmetry).

Thus, we can define a function ${ }^{29} h: R_{++}^{2} \times R_{+} \rightarrow R_{+}$

$$
h\left(x, a_{1}\right):=\left[\sup \left\{a_{2} \mid\left(a_{1}, a_{2}\right) \in V(x)\right\}\right]_{+} ;
$$

since $V(x)$ is comprehensive (Assumption 1), the supremum simply describes $\partial V(x)$. That is, for every $x \in R_{++}^{2} h(x, \cdot)$ is the function describing $\partial V(x)$ (its second coordinate $\left(a_{2}\right)$ as a function of the first $\left.\left(a_{1}\right)\right)$.

Given a function $a_{1}: R_{++}^{2} \rightarrow R_{+}$such that, for every $x \in R_{++}^{2}$, there exists $a_{2} \in R_{+}$for which $\left(a_{1}(x), a_{2}\right) \in \partial V(x)$, then we can define the functions $a_{2}: R_{++}^{2} \rightarrow R_{+}$and $^{30} \lambda: R_{++}^{2} \rightarrow \Delta_{++}^{2}$ by

$$
\begin{aligned}
& a_{2}(x):=h\left(x, a_{1}(x)\right) \\
& \lambda(x):=\left(\left(\frac{\left.\frac{\partial h}{\partial a_{1}}\right|_{\left(x, a_{1}(x)\right)}}{-\frac{x^{1}}{x^{2}}+\left.\frac{\partial h}{\partial a_{1}}\right|_{\left(x, a_{1}(x)\right)}}\right),\left(\frac{-\frac{x^{1}}{x^{2}}}{-\frac{x^{1}}{x^{2}}+\left.\frac{\partial h}{\partial a_{1}}\right|_{\left(x, a_{1}(x)\right)}}\right)\right) .
\end{aligned}
$$

Note that $\left.\frac{\partial h}{\partial a_{1}}\right|_{\left(x, a_{1}(x)\right)}<0$ in this range; hence $\lambda$ is well defined.

${ }^{29}$ In the region where $a_{1}>0$ and $h\left(x, a_{1}\right)>0$ the function $h$ is $C^{1}$. Since our discussion will be limited to an individually rational range, i.e., $a_{1} \geq r_{1}>0$ and $h\left(x, a_{1}\right) \geq r_{2}>0$, large values of $a_{1}$ (which cause $\sup \left\{a_{2} \mid\left(a_{1}, a_{2}\right) \in V(x)\right\}$ to be negative) will be excluded. Therefore, we can assume that $h$ is $C^{1}$.

${ }^{30}$ Recall, $\Delta_{++}^{2}$ is the interior of $\Delta^{2}$. 
Proposition 2. The above definitions are necessary and sufficient, to ensure that $a(x)=\left(a_{1}(x), a_{2}(x)\right)$ and $\lambda(x)$ satisfy the first two conditions of the consistent value.

Proof: We are looking for a potential consistent value $a$ such that the restriction to the first coordinate of $a(x)$ is $a_{1}(x)$. The first condition of the consistent value is $a(x) \in \partial V(x)$, so if $a(x)=\left(a_{1}(x), y\right)$, then

$$
y \in\left\{a_{2} \mid\left(a_{1}, a_{2}\right) \in \partial V(x)\right\},
$$

that is (from comprehensiveness, Assumption 1),

$$
y=\sup \left\{a_{2} \mid\left(a_{1}, a_{2}\right) \in V(x)\right\},
$$

and from the assumption restricting $a_{1}$ we can conclude ${ }^{31} y \geq 0$; hence

$$
y=h\left(x, a_{1}(x)\right) .
$$

The second condition of the consistent value states that $\lambda * x$ is the outward normal to the boundary of $V(x)$ at $a(x)$; the formula defining $\lambda$ provides precisely that, and is the only way of providing it. ${ }^{32}$

Let $a: R_{++}^{2} \rightarrow R_{++}^{2}$ be a smooth consistent value. With these definitions in mind, define $a_{1}: R_{++}^{2+} \rightarrow R_{++}$as the first coordinate of $a . a_{1}$ is continuously differentiable, and the third condition of the consistent value is equivalent to

$$
\left.\frac{\partial h}{\partial a_{1}}\right|_{\left(x, a_{1}(x)\right)}\left[x^{1} \frac{\partial a_{1}}{\partial x^{1}}(x)+x^{2} \frac{\partial a_{1}}{\partial x^{2}}(x)\right]=\left.x^{2} \frac{\partial h}{\partial x^{2}}\right|_{\left(x, a_{1}(x)\right)} \quad \forall x \in R_{++}^{2} .
$$

Lemma 3. Let $[2 ; V]$ be a continuum homogeneous game form with two types of players; then for each $x \in R_{++}^{2}$ and $c \in \partial V(x)$ :

$$
c=\left(c^{1}, c^{2}\right) \text { is in } \operatorname{core}(x, V) \text { if and only if } \frac{\partial h}{\partial x^{i}}\left(x, c^{1}\right)=0 \text {, for } i=1,2 \text {. }
$$

Proof: ${ }^{33}$ If $c=\left(c^{1}, c^{2}\right) \in \partial V(x)$ (that is, $\left.c^{2}=h\left(x, c^{1}\right)\right)$ is in the core of $(x, V)$, then for every $\varepsilon>0$ :

$$
c^{2} \geq h\left(x^{1}-\varepsilon, x^{2}, c^{1}\right) .
$$

Indeed, if there were $\varepsilon>0$ and $c^{\prime}>c^{2}$ such that $\left(c^{1}, c^{\prime}\right) \in \partial V\left(x^{1}-\varepsilon, x^{2}\right)$, then the sub-coalition $\left(x^{1}-\varepsilon, x^{2}\right)$ would contradict $\left(c^{1}, c^{2}\right)$ being in the core. Hence, as $h$ is differentiable,

$$
\frac{\partial h}{\partial x^{1}}\left(x, c^{1}\right)=\lim _{\varepsilon \rightarrow 0^{+}} \frac{h\left(x, c^{1}\right)-h\left(x-(\varepsilon, 0), c^{1}\right)}{\varepsilon} \geq 0 .
$$

${ }^{31}$ For every $x \in R_{++}^{2}$ there exists a non-negative $a_{2}$ such that $\left(a_{1}(x), a_{2}\right) \in \partial V(x) \subset V(x)$ and $y$ is the supremum of these values: therefore, $y$ is non-negative.

${ }^{32}$ By Assumption 4 there is a unique outward normal to the boundary of $V(x)$ at every point.

33 At first sight this lemma might seem to be simply a conclusion from Proposition 1, Equation 3 and the non-levelness assumption, but actually this lemma is stronger because no strict convexity (Assumptions 5 and 6 ) is needed here. 
And by the same argument

$$
\frac{\partial h}{\partial x^{2}}\left(x, c^{1}\right) \geq 0
$$

Recall (from Assumption 7) that $h\left(x, c^{1}\right)=h\left(t x, c^{1}\right)$ for all $t>0$ and any $c^{1}$; therefore, by Euler's equation,

$$
x^{1} \frac{\partial h}{\partial x^{1}}\left(x, c^{1}\right)+x^{2} \frac{\partial h}{\partial x^{2}}\left(x, c^{1}\right)=0
$$

implying that

$$
\frac{\partial h}{\partial x^{i}}\left(x, c^{1}\right)=0, \quad \forall i
$$

On the other hand, if $c=\left(c^{1}, c^{2}\right) \notin \operatorname{core}(x, V)$, then there exists $x^{*} \in R_{++}^{2}$ such that $x^{*} \leq x$, and $d \in V\left(x^{*}\right)$ such that $c<d$.

Without loss of generality we can assume ${ }^{34} x^{*}=x-\left(\varepsilon_{0}, 0\right)$. Since $c<d$, we can conclude from comprehensiveness and non-levelness (Assumptions 1 and 3) that $c \in V\left(x^{*}\right) \backslash \partial V\left(x^{*}\right)$. Therefore

$$
h\left(x^{*}, c^{1}\right)=c^{*}=c^{2}+\delta,
$$

for some $\delta>0$. Recall that

$$
h\left(x, c^{1}\right)=c^{2}
$$

hence from Assumptions 2 and 7 (homogeneity) we get, for every $\varepsilon$ such that $0<\varepsilon<\varepsilon_{0}$ :

$$
h\left(x-(\varepsilon, 0), c^{1}\right) \geq c^{2}+\frac{\delta}{\varepsilon_{0}} \varepsilon .
$$

Since $h$ is differentiable,

$$
\frac{\partial h}{\partial x^{1}}\left(x, c^{1}\right)=\lim _{\varepsilon \rightarrow 0^{+}} \frac{h\left(x, c^{1}\right)-h\left(x-(\varepsilon, 0), c^{1}\right)}{\varepsilon} \leq \lim _{\varepsilon \rightarrow 0^{+}} \frac{-\frac{\delta}{\varepsilon_{0}} \varepsilon}{\varepsilon}=-\frac{\delta}{\varepsilon_{0}} .
$$

That is,

$$
\frac{\partial h}{\partial x^{1}}\left(x, c^{1}\right) \neq 0
$$

To conclude, note that:

34 Because of homogeneity (Assumption 7), and possibly exchanging between the 2 coordinates. 
1. Assumption 3 (non-levelness) guarantees that $\left.\frac{\partial h}{\partial a_{1}}\right|_{\left(x, a_{1}(x)\right)} \neq 0, \infty$ in the relevant range.

2. For every $x \in R_{++}^{2}, a(x)=\left(a_{1}(x), h\left(x, a_{1}(x)\right)\right) \in \operatorname{core}(x, V)$ if and only if $\frac{\partial h}{\partial x^{2}}\left(x, a_{1}(x)\right)=0$.

3. For $a_{1}$ which is $C^{1}: a_{1}$ is homogeneous of degree 0 is equivalent to $\sum_{i} x^{i} \frac{\partial a_{1}}{\partial x^{i}}(x)=0$, i.e., $\left.\frac{d a_{1}(t x)}{d t}\right|_{t=1}=0$.

\section{The main result}

The main result of this paper consists of the characterization of all individually rational smooth $\left(C^{1}\right)$ consistent values, for games with a continuum of players of two types.

Theorem 4. Let $[2 ; V]$ be an NTU homogeneous game form with a continuum of players. Then every smooth individually rational consistent value a of $[2 ; V]$ belongs to the ConvCore (i.e., $a(x) \in \operatorname{ConvCore}(x, V)$ for all $\left.x \in R_{++}^{2}\right)$.

Furthermore, any $C^{1}$ function $g: \Delta_{++}^{2} \rightarrow R_{++}^{2}$, such that $g(x) \in$ ConvCore $(x, V)$ for all $x$ in $\Delta_{++}^{2}$, can be uniquely extended to an individually rational smooth consistent value.

That is, on the interior of $\Delta^{2}$ take an arbitrary $C^{1}$ selection from the ConvCore and extend it to an individually rational smooth consistent value.

Notice that this theorem does not imply that such a consistent value must exist. An example where no individually rational smooth consistent value exists can be found in Section 7.2.

By Theorem 4 we get, in particular, that when the ConvCore and the core are not equal (i.e., there exists $x \in R_{++}^{2} \operatorname{such}_{\text {that }}{ }^{35} \operatorname{core}(x, V) \neq$ ConvCore $(x, V))$ there is no equivalence between the consistent value and the core. Furthermore, there may not be any consistent value which belongs to the core. Still we have:

Theorem 5. Let $[2 ; V]$ be an NTU homogeneous game form with a continuum of players, and let $a$ be an individually rational smooth consistent value of $[2 ; V]$.

For every coalition profile $x$ in $R_{++}^{n}$, the limit of $a(t x)$, as $t$ tends to infinity, exists and is in $\operatorname{core}(x, V)$ (i.e., $\lim _{t \rightarrow \infty} a(t x) \in \operatorname{core}(x, V)$ ), and so does the limit as t tends to zero (i.e., $\lim _{t \rightarrow 0} a(t x) \in \operatorname{core}(x, V)$ ).

That is, for every relative mass of players, ${ }^{36}$ the limit, as the total mass of all players tends to 0 or to $\infty$, belongs to the core.

The rest of the paper will be devoted to proving these two theorems. We start by proving the first part of Theorem 4:

Proposition 6. Let $[2 ; V]$ be an NTU homogeneous game form with a continuum of players, and $a: R_{++}^{2} \rightarrow R_{++}^{2}$ an individually rational smooth consistent value

\footnotetext{
${ }^{35}$ It is always true that $\operatorname{core}(x, V) \subseteq \operatorname{ConvCore}(x, V)$; therefore, $\operatorname{core}(x, V) \neq \operatorname{ConvCore}(x, V)$ means that there are points in ConvCore $(x, V) \backslash \operatorname{core}(x, V)$.

36 That is, for every proportion $\alpha \in(0,1)$ of $\frac{x^{1}}{x^{2}}$.
} 


$$
\mathrm{V}\left(\mathrm{x}^{*}\right)
$$

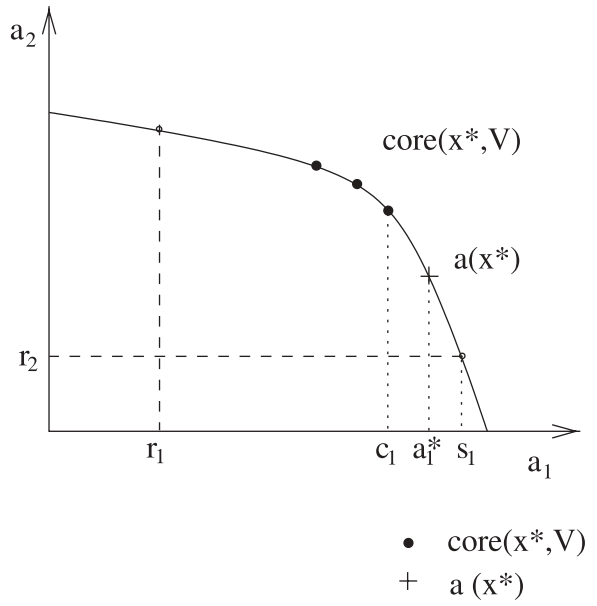

Fig. 2. Description of $V\left(x^{*}\right)$

of this game. Then a belongs to the ConvCore (i.e., $a(x) \in \operatorname{ConvCore}(x, V)$ for every $x \in R_{++}^{2}$ ).

Proof: Define $a_{1}: R_{++}^{2} \rightarrow R_{++}$as the projection of $a$ on the first coordinate. Since $a$ is a consistent value, $a(x) \in \partial V(x)$; that is:

$$
a(x)=\left(a_{1}(x), h\left(x, a_{1}(x)\right)\right) \quad \forall x \in R_{++}^{2} .
$$

Assume to the contrary that $a$ does not belong to the ConvCore. Then, there exists $x^{*} \in R_{++}^{2}$ such that:

$$
\left(a_{1}\left(x^{*}\right), h\left(x, a_{1}\left(x^{*}\right)\right)\right) \notin \operatorname{ConvCore}\left(x^{*}, V\right) .
$$

Assume, without loss of generality, ${ }^{37}$ that $b^{1}<a_{1}\left(x^{*}\right)$ for any $b \in \operatorname{core}\left(x^{*}, V\right)$. Denote $c_{1}$ the first coordinate of the point in core $\left(x^{*}, V\right)$ closest $^{38}$ to $a\left(x^{*}\right)$ along $\partial V\left(x^{*}\right)$. See Figure 2 .

We trace the consistent value along the ray $\left\{t x^{*} \mid t>0\right\}$. Note that $V\left(t x^{*}\right)$, core $\left(t x^{*}, V\right)$ and the individually rational values $r_{1}, r_{2}$ are independent of $t$.

Now, the third consistent value condition is equivalent (see Equation 3 ) to the assertion that for every $t_{0}>0$

$$
\left.\frac{d a_{1}\left(t x^{*}\right)}{d t}\right|_{t=t_{0}}=\frac{1}{t_{0}} \frac{\left.x^{2} \frac{\partial h}{\partial x^{2}}\right|_{\left(t_{0} x^{*}, a_{1}\left(t_{0} x^{*}\right)\right)}}{\left.\frac{\partial h}{\partial a_{1}}\right|_{\left(t_{0} x^{*}, a_{1}\left(t_{0} x^{*}\right)\right)}} .
$$

${ }^{37}$ If this were not so (i.e., $\left.a_{1}\left(x^{*}\right)<b^{1}\right)$, then $b^{2}<a_{2}\left(x^{*}\right)$ for any $b \in \operatorname{core}\left(x^{*}, V\right)$, this is true because $a(x)$ is efficient thus one type of player must be better of then in the core. In this case we would interchange the coordinates.

${ }^{38}$ If core $\left(x^{*}, V\right)$ is not closed, then the closest point in its closure. If core $\left(x^{*}, V\right)$ is empty, then any efficient individually rational point whose first coordinate is smaller then $a_{1}\left(x^{*}\right)$ will do. 
Denote $a_{1}^{*}=a_{1}\left(x^{*}\right)$ and let $s_{1}$ be such that ${ }^{39}\left(s_{1}, r_{2}\right) \in \partial V\left(x^{*}\right)$. Then ${ }^{40}$

$c_{1}<a_{1}^{*} \leq s_{1}$.

Notice that:

- For $a \in\left[a_{1}^{*}, s_{1}\right] \quad x^{* 2} \frac{\partial h}{\partial x^{2}}\left(x^{*}, a\right) \neq 0$, since only for points in the core does this equal 0 , and no core point is in the range $\left[a_{1}^{*}, s_{1}\right]$. Therefore, $x^{* 2} \frac{\partial h}{\partial x^{2}}\left(x^{*}, a\right) \geq \delta$ for every $a \in\left[a_{1}^{*}, s_{1}\right]$, for some ${ }^{41} \delta>0$.

- From the assumptions of convexity and non-levelness (Assumptions 1 and $3)$, there exist $d_{0}, d_{1}>0$ such that for every $a \in\left[a_{1}^{*}, s_{1}\right]$ :

$$
-d_{1} \leq\left.\frac{\partial h}{\partial a_{1}}\right|_{\left(t x^{*}, a\right)}=\left.\frac{\partial h}{\partial a_{1}}\right|_{\left(x^{*}, a\right)} \leq-d_{0}
$$

(the values are bounded between $\left.\frac{\partial h}{\partial a_{1}}\right|_{\left(x^{*}, c_{1}\right)}$ and $\left.\frac{\partial h}{\partial a_{1}}\right|_{\left(x^{*}, s_{1}\right)}$, which are both negative $\left.^{42}\right)$.

Hence, for every $t_{0}>0$ :

$$
-\left.\frac{d a_{1}\left(t x^{*}\right)}{d t}\right|_{t=t_{0}}=\frac{1}{t_{0}} \frac{\left.x^{2} \frac{\partial h}{\partial x^{2}}\right|_{\left(t_{0} x^{*}, a_{1}\left(t_{0} x^{*}\right)\right)}}{-\left.\frac{\partial h}{\partial a_{1}}\right|_{\left(t_{0} x^{*}, a_{1}\left(t_{0} x^{*}\right)\right)}} \geq \frac{1}{t_{0}} \frac{\delta}{d_{1}},
$$

which, in turn, implies that

$$
\left.\int_{1}^{0} \frac{d a_{1}\left(t x^{*}\right)}{d t}\right|_{t=t_{0}} d t_{0}=\int_{0}^{1}-\left.\frac{d a_{1}\left(t x^{*}\right)}{d t}\right|_{t=t_{0}} d t_{0} \geq \frac{\delta}{d_{1}} \int_{0}^{1} \frac{1}{t_{0}} d t_{0}=\infty .
$$

Therefore, there exists $0<t^{\prime} \leq 1$ for which $a_{1}\left(t^{\prime} x^{*}\right)=s_{1}$ (the end of the region where our estimates hold). Clearly, $\left.\frac{d a_{1}\left(t x^{*}\right)}{d t}\right|_{t=t^{\prime}}<0$. Then, there exists $0<t^{\prime \prime}<t^{\prime}$ for which $a_{1}\left(t^{\prime \prime} x^{*}\right)<s_{1}$. This contradicts individual rationality. ${ }^{43}$

Thus our original assumption is not valid.

In the next lemma we only consider one profile $x$ (more precisely, the ray $\{t x \mid t>0\}$ through $x$ ). That is, the lemma is valid for every profile $x$ independently of other profiles.

Lemma 7. Let $[2 ; V]$ be an NTU homogeneous game form with a continuum of players, let $a: R_{++}^{2} \rightarrow R_{++}^{2}$ be an individually rational smooth consistent value of this game, and let $x^{*} \in R_{++}^{2}$ be a coalition profile. If $a\left(x^{*}\right)$ is in

\footnotetext{
39 Just as in the definition of $h$, there is a single point satisfying this condition.

${ }^{40}$ Since $a$ is individually rational $a_{1}^{*} \leq s_{1}$.

${ }^{41}$ It is verifiable that in this range $\frac{\partial h}{\partial x^{2}}\left(x^{*}, a\right)$ is indeed positive, but it is of no consequence. Since the function is continuous and does not assume the value 0 in the range, then it is either positive or negative in the entire range. If it were negative in the whole range, the same type of arguments as in the rest of this proof would hold when $t$ tends to infinity, instead of to zero.

If the core was empty this might indeed be necessary, or else we would need to interchange the coordinates.

42 The negative values are due to Assumption $1(V(x)$ being comprehensive).

${ }^{43}$ Evidently, $h\left(x^{*}, a_{1}\left(t^{\prime \prime} x^{*}\right)\right)$ is not individually rational for players of type 2 .
} 
$\operatorname{core}\left(x^{*}, V\right)$ then $a\left(t x^{*}\right)$ is independent of $t$ (i.e., for every $t>0 \quad a\left(x^{*}\right)=$ $\left.a\left(t x^{*}\right) \in \operatorname{core}\left(t x^{*}, V\right)\right)$.

Proof: Since $a\left(x^{*}\right) \in \operatorname{core}\left(x^{*}, V\right)$, by Lemma $\left.3 \frac{\partial h}{\partial x^{2}}\right|_{\left(x^{*}, a_{1}\left(x^{*}\right)\right)}=0$.

Recall (Equation 4) that for every $t_{0}>0$

$$
\left.\frac{d a_{1}\left(t x^{*}\right)}{d t}\right|_{t=t_{0}}=\frac{1}{t_{0}} \frac{\left.x^{2} \frac{\partial h}{\partial x^{2}}\right|_{\left(t_{0} x^{*}, a_{1}\left(t_{0} x^{*}\right)\right)}}{\left.\frac{\partial h}{\partial a_{1}}\right|_{\left(t_{0} x^{*}, a_{1}\left(t_{0} x^{*}\right)\right)}}
$$

Therefore, for every $t_{0}>0$ such that $a\left(t_{0} x^{*}\right) \in \operatorname{core}\left(x^{*}, V\right)$ :

$$
\left.\frac{d a_{1}\left(t x^{*}\right)}{d t}\right|_{t=t_{0}}=0
$$

Since this is true for $t_{0}=1$, it is also true for every $t_{0}>0$. That is,

$$
a_{1}\left(t_{0} x^{*}\right)=a_{1}\left(x^{*}\right) \quad \forall t_{0}>0 .
$$

\section{The case of a unique core point}

Proposition 8. If for each $x \in R_{++}^{2}$ the core of $(x, V)$ consists of a single point, and $a: R_{++}^{2} \rightarrow R_{++}^{2}$ is an individually rational smooth consistent value of this game, then a coincides with the core, i.e.,

$$
\{a(x)\}=\operatorname{core}(x, V) \quad \forall x \in R_{++}^{2} .
$$

Proof: Assume to the contrary that $a$ does not coincide with the core. Then, there exists $x^{*} \in R_{++}^{2}$ such that:

$$
\left(a_{1}\left(x^{*}\right), h\left(x, a_{1}\left(x^{*}\right)\right)\right) \notin \operatorname{core}\left(x^{*}, V\right) .
$$

Since there is a unique core point, the ConvCore is precisely the core; thus

$$
\left(a_{1}\left(x^{*}\right), h\left(x, a_{1}\left(x^{*}\right)\right)\right) \notin \operatorname{ConvCore}\left(x^{*}, V\right) .
$$

And by Proposition 6 our original assumption is not valid.

Remark: If we do not demand that the consistent value be smooth $\left(C^{1}\right)$, and consider also semi-smooth consistent values, we discover that these do not coincide with the core even for a game with a unique core point.

Example 1. For the game with two types of players, and two commodities with utility functions: ${ }^{44}$

\footnotetext{
44 The following game does not satisfy Assumption 3 (non-levelness). This problem can be solved by replacing the utility function with$$
u^{i}\left(w_{1}, w_{2}\right)=\sqrt{w_{1}}+\sqrt{w_{2}}-\varepsilon \quad \forall i,
$$

for some small $\varepsilon>0$. This does not change the validity of the example at all; it only complicates some of the calculations.
} 


$$
u^{i}\left(w_{1}, w_{2}\right)=\sqrt{w_{1}}+\sqrt{w_{2}} \quad \forall i
$$

and initial endowment vectors

$$
e^{1}=(1,0), \quad e^{2}=(0,1),
$$

the game function is:

$$
V(x)=\left\{a \in R_{+}^{2} \mid x^{1}\left(a^{1}\right)^{2}+x^{2}\left(a^{2}\right)^{2} \leq\left(\sqrt{x^{1}}+\sqrt{x^{2}}\right)^{2}\right\}-R_{+}^{2} .
$$

Therefore,

$$
h\left(x, a_{1}\right)=\sqrt{\frac{x^{1}+x^{2}+2 \sqrt{x^{1} x^{2}}-x^{1}\left(a_{1}\right)^{2}}{x^{2}}} ;
$$

hence

$$
\left.\frac{\partial h\left(x, a_{1}\right)}{\partial x^{2}}\right|_{\left(x, a_{1}(x)\right)}=\frac{-\frac{x^{1}}{x^{2}}-\sqrt{\frac{x^{1}}{x^{2}}}+\left(a_{1}(x)\right)^{2} \frac{x^{1}}{x^{2}}}{2 h\left(x, a_{1}(x)\right) x^{2}}
$$

and

$$
\left.\frac{\partial h\left(x, a_{1}\right)}{\partial a_{1}}\right|_{\left(x, a_{1}(x)\right)}=\frac{-2 \frac{x^{1}}{x^{2}} a_{1}(x)}{2 h\left(x, a_{1}(x)\right)} .
$$

We need to solve:

$$
\left.t_{0} \frac{d a_{1}(t x)}{d t}\right|_{t=t_{0}}=\frac{\left.x^{2} \frac{\partial h\left(x, a_{1}\right)}{\partial x^{2}}\right|_{\left(t_{0} x, a_{1}\left(t_{0} x\right)\right)}}{\left.\frac{\partial h\left(x, a_{1}\right)}{\partial a_{1}}\right|_{\left(t_{0} x, a_{1}\left(t_{0} x\right)\right)}}=\frac{1+\sqrt{\frac{x^{2}}{x^{1}}}-\left(a_{1}\left(t_{0} x\right)\right)^{2}}{2 a_{1}\left(t_{0} x\right)} .
$$

One possible solution is the homogeneous solution (which coincides with the core), $\frac{d a_{1}(t x)}{d t}=0$. From this equation we get the unique consistent value

$$
a_{1}(x)=\operatorname{core}_{1}(x, V)=\sqrt{1+\sqrt{\frac{x^{2}}{x^{1}}}} .
$$

But, other possible solutions (which are not homogeneous) can be derived by manipulating the differential equation. We get

$$
\forall t_{0}>\left.0 \quad 2 t_{0} a_{1}\left(t_{0} x\right) \frac{d a_{1}(t x)}{d t}\right|_{t=t_{0}}=1+\sqrt{\frac{x^{2}}{x^{1}}}-\left(a_{1}\left(t_{0} x\right)\right)^{2},
$$

which can be solved by

$$
\forall x \in \Delta_{++}^{2}, \quad \forall t_{0}>0 \quad a_{1}\left(t_{0} x\right)=\sqrt{1+\sqrt{\frac{x^{2}}{x^{1}}}-\frac{1}{t_{0}}} .
$$


By using the normalization $t_{0}=\left(x^{1}+x^{2}\right)$, and truncating the proposed function so that it will be individually rational we can get the semi-smooth consistent value ${ }^{45}$

$$
a_{1}(x):=\left\{\begin{array}{ll}
\sqrt{1+\sqrt{\frac{x^{2}}{x^{1}}}-\frac{1}{\left(x^{1}+x^{2}\right)}} & \text { if }\left(x^{1}+x^{2}\right)>1 \\
\sqrt{1+\sqrt{\frac{x^{2}}{x^{1}}}} & \text { otherwise. }
\end{array} .\right.
$$

- The function is not $C^{1}$; furthermore, it is not even continuous along the line $\left(x^{1}+x^{2}\right)=1$, but it is differentiable from below.

- The function $a: R_{++}^{2} \rightarrow R_{++}^{2}$ defined by $a(x):=\left(a_{1}(x), h\left(x, a_{1}(x)\right)\right)$ is a semismooth consistent value for this game.

- This semi-smooth consistent value does not coincide with the $\operatorname{core}^{46}$ (since $a(x) \neq \operatorname{core}(x, V)$ for all $x \in R_{++}^{2}$ such that $\left.\left(x^{1}+x^{2}\right)>1\right)$.

As this example shows, allowing for semi-smooth consistent values enlarges the set of consistent values to be considered. Thus, this extension not only does not contribute to a possible equivalence with the core but, in fact, it shows that semi-smooth consistent values offer a greater selection of consistent values differing from the core.

\section{Proof of main theorem}

\subsection{Flow of the consistent value}

When considering consistent values which are smooth $\left(C^{1}\right)$, we can trace, for each $x \in \Delta_{++}^{2}$, the movement of the consistent value as the profile moves along the ray $\{t x \mid t>0\}$.

From our assumptions $V(t x)=V(x)$ for all $t>0$; hence $h(t x, \cdot)=h(x, \cdot)$ for all $t>0$. Recall (Equation 4) that the consistent value requirements are equivalent (in this case) to:

$$
\left.\frac{d a_{1}(t x)}{d t}\right|_{t=t_{0}}=\frac{1}{t_{0}} \frac{\left.x^{2} \frac{\partial h\left(x, a_{1}\right)}{\partial x^{2}}\right|_{\left(t_{0} x, a_{1}\left(t_{0} x\right)\right)}}{\left.\frac{\partial h\left(x, a_{1}\right)}{\partial a_{1}}\right|_{\left(t_{0} x, a_{1}\left(t_{0} x\right)\right)}} \quad \forall t_{0}>0 .
$$

Let $a: R_{++}^{2} \rightarrow R_{++}^{2}$ be a smooth consistent value, and let $x^{*} \in R_{++}^{2}$. If the core of $\left(x^{*}, V\right)$ has a unique point $c \in \partial V(x)$, then either (see Proposition 8):

1. There exists a $t_{0}>0$ such that $a_{1}\left(t_{0} x^{*}\right)=c^{1}$, which implies $a_{1}\left(t x^{*}\right)=c^{1}$ for all $t>0$; or

2. For all $t>0, \quad a_{1}\left(t x^{*}\right) \neq c^{1}$, which implies that there exists a $t_{0}>0$ such that $\left(a_{1}\left(t_{0} x^{*}\right), h\left(x^{*}, a_{1}\left(t_{0} x^{*}\right)\right)\right)$ is not individually rational.

\footnotetext{
${ }^{45}$ See remark after Definition 6 in Section 3.2.

${ }^{46}$ Recall, $\operatorname{core}(x, V)=\left(\sqrt{1+\sqrt{\frac{x^{2}}{x^{1}}}}, \sqrt{1+\sqrt{\frac{x^{1}}{x^{2}}}}\right)$.
} 


$$
\hat{V}\left(t^{*}\right)=t \hat{V}\left(x^{*}\right)
$$

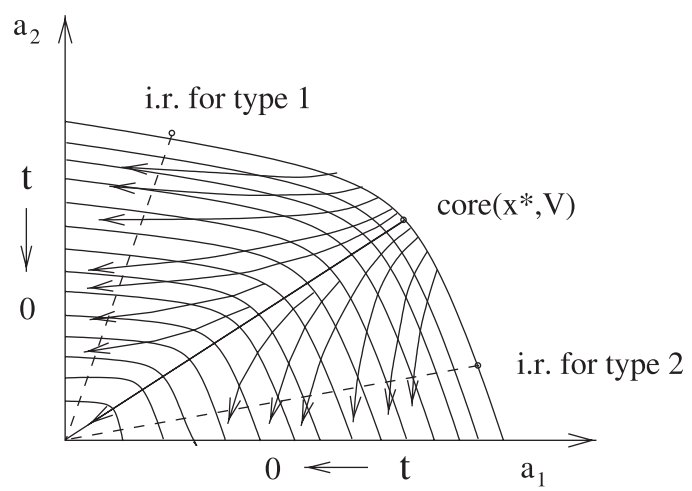

Fig. 3. Flow of the consistent value (a unique point in the core)

That is, if we look at $\left(t a_{1}\left(t x^{*}\right), t h\left(t x^{*}, a_{1}\left(t x^{*}\right)\right)\right)$ derived from a smooth consistent value, then its value is determined by the value $a_{1}\left(x^{*}\right)$ (Figure 3 ). The figure shows the boundary of $\hat{V}\left(t x^{*}\right)$ for different values of $t$. Since $V\left(t x^{*}\right)=$ $V\left(x^{*}\right)$ for all $t>0, \hat{V}\left(t x^{*}\right)=t \hat{V}\left(x^{*}\right)$. Thus the boundary line is lower as $t$ decreases.

Now assume that the core of $\left(x^{*}, V\right)$ does not consist of a unique point. ${ }^{47}$ Define $c_{\max }^{1}=\sup _{c \in \operatorname{core}\left(x^{*}, V\right)}\left(c^{1}\right)$ and $c_{\min }^{1}=\inf _{c \in \operatorname{core}\left(x^{*}, V\right)}\left(c^{1}\right)$ Let $a: R_{++}^{2} \rightarrow R_{++}^{2}$ be a smooth consistent value; then either:

1. There exist $t_{0}>0$ and $c \in \operatorname{core}\left(x^{*}, V\right)$ such that $a_{1}\left(t_{0} x^{*}\right)=c^{1}$. This implies that $a_{1}\left(t x^{*}\right)=c^{1}$ for all $t>0$ (see Lemma 7).

2. There exists a $t_{0}>0$ such that either ${ }^{48} a_{1}\left(t_{0} x^{*}\right)<c_{\min }^{1}$ or $a_{1}\left(t_{0} x^{*}\right)>c_{\max }^{1}$. This implies that for all $t>0$, either $a_{1}\left(t x^{*}\right)<c_{\min }^{1}$ or $a_{1}\left(t x^{*}\right)>c_{\max }^{1}$ (respectively), which in turn implies that there exists a $t_{1}>0$ such that $\left(a_{1}\left(t_{1} x^{*}\right), h\left(x^{*}, t_{1} x^{*}\right)\right)$ is not individually rational (see Proposition 6).

3. There exists a $t_{0}>0$ such that $c_{\min }^{1}<a_{1}\left(t_{0} x^{*}\right)<c_{\max }^{1}\left(\right.$ and $a_{1}\left(t_{0} x^{*}\right) \neq c^{1}$ for all $\left.c \in \operatorname{core}\left(x^{*}, V\right)\right)$. This implies that $c_{\min }^{1}<a_{1}\left(t x^{*}\right)<c_{\max }^{1}$ for all $t>0$ (and $a_{1}\left(t x^{*}\right) \neq c^{1}$ for all $c \in \operatorname{core}\left(x^{*}, V\right)$ ). Where does this lead?

If we look again at $\left(t a_{1}\left(t x^{*}\right), t h\left(t x^{*}, a_{1}\left(t x^{*}\right)\right)\right)$ derived from this smooth consistent value, then its value is determined by the value $a_{1}\left(x^{*}\right)$ (Figure 4). Again, the figure shows the boundary of $\hat{V}\left(t x^{*}\right)$ for different values of $t$.

If there were three points in core $\left(x^{*}, V\right)$, a reasonable assumption ${ }^{49}$ for the behavior of $a_{1}\left(t x^{*}\right)$ in the last case would be:

\footnotetext{
${ }^{47}$ This does not mean that there is a non-unique core point for every $x \in R_{++}^{2}$, but that for $x^{*} \in R_{++}^{2}$ the core consists of more than a single point $\left(\left|\operatorname{core}\left(x^{*}, V\right)\right|>1\right)$.

${ }^{48}$ If $c_{\min }$ in not in the core but only in its closure then $<$ should be replaced by $\leq$. The same is true for $c_{\max }$, with $>$ replaced by $\geq$.

49 This assumption arises from the continuity of $x^{* 2} \frac{\partial h}{\partial x^{2}}\left(x^{*}, a\right)$, in the same manner as in Proposition 6 .
} 


$$
\hat{\mathrm{V}}\left(\mathrm{tx}^{*}\right)=\mathrm{t} \hat{\mathrm{V}}\left(\mathrm{x}^{*}\right)
$$

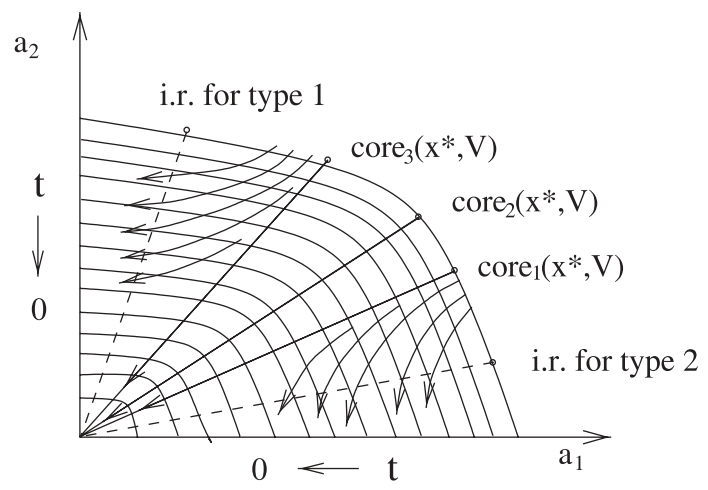

Fig. 4. Flow of the consistent value (three points in the core)

$$
\lim _{t \rightarrow 0} a_{1}\left(t x^{*}\right)=c_{2}^{1}
$$

and:

$$
\lim _{t \rightarrow \infty} a_{1}\left(t x^{*}\right)=c_{1}^{1} \text { or } c_{3}^{1} .
$$

An example of this behavior can be found in Section 7.1.

\subsection{The dynamic approach}

As we have seen, the projection $a_{1}$ on the first coordinate of an individually rational smooth consistent value $a: R_{++}^{2} \rightarrow R_{++}^{2}$ satisfies:

$$
t \frac{d a_{1}}{d t}=\frac{x^{2} \frac{\partial h}{\partial x^{2}}}{\frac{\partial h}{\partial a_{1}}}
$$

with $t=\left(x^{1}+x^{2}\right)$ and $h$ dependent of $a_{1}$ and $\frac{x^{1}}{x^{2}}$ only. Transforming our equations to functions of $T:=\log t=\log \left(x^{1}+x^{2}\right)$ and $\alpha:=\frac{x^{1}}{x^{1}+x^{2}}$ we get:

$$
\frac{d a_{1}}{d T}(\alpha, T)=-\frac{\left.\alpha(1-\alpha) \frac{\partial h}{\partial \alpha}\right|_{\left((\alpha, 1-\alpha), a_{1}\right)}}{\left.\frac{\partial h}{\partial a_{1}}\right|_{\left((\alpha, 1-\alpha), a_{1}\right)}} .
$$

Thus, the movement of the consistent value along the "time" axis $\left(\log \left(x^{1}+x^{2}\right)\right)$, for every given $\alpha\left(=\frac{x^{1}}{x^{1}+x^{2}}\right)$, can be described as a timeindependent dynamical system. That is, a movement which satisfies the equation: 


$$
z^{\prime}=f(z)
$$

for

$$
f\left(\alpha, a_{1}\right):=\left(0, \frac{d a_{1}}{d T}(\alpha, T)\right)=\left(0,-\frac{\left.\alpha(1-\alpha) \frac{\partial h}{\partial \alpha}\right|_{\left((\alpha, 1-\alpha), a_{1}\right)}}{\left.\frac{\partial h}{\partial a_{1}}\right|_{\left((\alpha, 1-\alpha), a_{1}\right)}}\right) .
$$

If we further assume that $h$ is $C^{2}$, then from non-levelness (Assumption 3) we get that $f$ is $C^{1}$.

Proposition 9 (Note that this is exactly the second part of Theorem 4.)

Let $[2 ; V]$ be a continuum homogeneous game form with two types of players that satisfies our assumptions. If $g: \Delta_{++}^{2} \rightarrow R_{++}^{2}$ is a $C^{1}$ function, such that:

$$
g(x) \in \operatorname{ConvCore}(x, V) \quad \forall x \in \Delta_{++}^{2},
$$

then there exists a unique individually rational smooth consistent value $a: R_{++}^{2} \rightarrow R_{++}^{2}$ for this game which extends g. I.e., such that:

$$
a(x)=g(x) \quad \forall x \in \Delta_{++}^{2} .
$$

Proof: For the function $f:(0,1) \times R_{++} \rightarrow R^{2}$ defined by

$$
f\left(\alpha, a_{1}\right):=\left(0,-\frac{\left.\alpha(1-\alpha) \frac{\partial h}{\partial \alpha}\right|_{\left((\alpha, 1-\alpha), a_{1}\right)}}{\left.\frac{\partial h}{\partial a_{1}}\right|_{\left((\alpha, 1-\alpha), a_{1}\right)}}\right),
$$

we want to solve: ${ }^{50}$

$$
\begin{aligned}
& z^{\prime}=f(z) \\
& z(0)=\left(\alpha_{0}, \pi_{1}\left(g\left(\alpha_{0}, 1-\alpha_{0}\right)\right)\right),
\end{aligned}
$$

for every $\alpha_{0} \in(0,1)$.

For every $\left(\alpha^{*}, a^{*}\right) \in(0,1) \times R_{++}$there exists a range $l=l\left(\alpha^{*}, a^{*}\right)>0$, and a single solution $z:(-l, l) \rightarrow(0,1) \times R_{++}$to this equation (see [6, pp. 162169]).

Define $B:=\{\alpha \in(0,1) \mid \operatorname{core}((\alpha, 1-\alpha), V)$ has more than one point $\}$, and let $A \subset(0,1) \times R_{++}$be the following compact ${ }^{51}$ set: $^{52}$

$$
A:=\left\{(\alpha, a) \mid \begin{array}{c}
\alpha \in B^{c} \\
a \in \pi_{1}(\operatorname{ConvCore}((\alpha, 1-\alpha), V))
\end{array}\right\} .
$$

${ }^{50} \pi_{1}$ is the projection, on the first coordinate, of the value.

51 The compactness of this set is assured because of:

- the compactness of the first coordinate;

- the continuity of the core (as a function of $\alpha$ ).

$52 B^{c}$ is the closure of the set $B . \pi_{1}$ is the projection, on the first coordinate, of the value. 
Since every $(\alpha, a)$ such that $a \in \pi_{1}(\operatorname{core}((\alpha, 1-\alpha), V))$ satisfies $^{53} f(\alpha, a)=$ $(0,0)$, it follows that a solution $z$ starting inside A stays inside A. ${ }^{54}$

From this we can deduce that there exists a solution $z:(-\infty, \infty) \rightarrow A \subset$ $(0,1) \times R_{++}$to our equation (see [6, pp. 172-173]).

Define a dynamic system $\phi:\left\{\left(T, z^{*}\right) \mid T \in R, z^{*} \in A\right\} \rightarrow R^{2}$ by:

$\phi\left(T, z^{*}\right):=z(T)$,

where $z: R \rightarrow A$ is the unique solution to

$$
\begin{aligned}
& z^{\prime}=f(z) \\
& z(0)=z^{*} .
\end{aligned}
$$

This $\phi$ is $C^{1}$ (see [6, pp. 175-176, 302]).

Now, define $a_{1}: R_{++}^{2} \rightarrow R_{+}$by $^{55}$

$$
a_{1}(x)=\pi_{2}\left(\phi\left(\log \left(x^{1}+x^{2}\right),\left(\frac{x^{1}}{x^{1}+x^{2}}, \pi_{1}(g(x))\right)\right)\right),
$$

and define

$$
a(x)=\left(a_{1}(x), h\left(x, a_{1}(x)\right)\right) .
$$

Evidently,

1. The function $a$ is $C^{1}$.

2. For every $x \in \Delta_{++}^{2}$

$$
\pi_{1}(a(x))=a_{1}(x)=\pi_{2}\left(\phi\left(0,\left(x^{1}, \pi_{1}(g(x))\right)\right)\right)=\pi_{2}\left(x^{1}, \pi_{1}(g(x))\right)=\pi_{1}(g(x)) ;
$$

therefore (since both are in $\partial V(x))$

$$
a(x)=g(x) \quad \forall x \in \Delta_{++}^{2} .
$$

3. $a$ is a consistent value for this game (from the constraints which defined the dynamical system).

4. $a$ belongs to the ConvCore; therefore it is individually rational.

5. There exists no other consistent value $a^{\prime}(\neq a)$ for this game which extends $g$ (since the consistent-value conditions defined the dynamic system, and forced the extension of $g$ to $a$ ).

Corollary 10. There exists an individually rational smooth consistent value which belongs to the core if and only if there exists a $C^{1}$ function $g: \Delta_{++}^{2} \rightarrow R_{++}^{2}$, such that:

$$
g(x) \in \operatorname{core}(x, V) \quad \forall x \in \Delta_{++}^{2} .
$$

53 This means that positions on the boundary of $A$ are static in the dynamic motion.

54 I.e., $z(0) \in A^{\circ} \Rightarrow$ for all $l_{0} \in(-l, l), z\left(l_{0}\right) \in A^{\circ}$.

55 The value of this expression is positive, since the range of $\phi$ is $A \subset(0,1) \times R_{++} . \pi_{2}$ is the projection, on the second coordinate, of the value. 
It is important to note that in many cases no such $C^{1}$ selection from the core exists, even in game forms where there is an infinite selection of $C^{1}$ selections from the ConvCore.

One obvious question arises at this point, what does this consistent value tend to as the total mass of all players tends to 0 or to $\infty$. To answer this, let us now restate and prove Theorem 5 .

Theorem 5. Let $[2 ; V]$ be an NTU homogeneous game form with a continuum of players, which satisfies our assumptions. If $a: R_{++}^{2} \rightarrow R_{++}^{2}$ is a smooth consistent value for this game, then:

For every coalition profile $x$ in $R_{++}^{n}$, the limit of $a(t x)$, as t tends to infinity, exists and is in $\operatorname{core}(x, V)$ (i.e., $\lim _{t \rightarrow \infty} a(t x) \in \operatorname{core}(x, V)$ ), and so does the limit as $t$ tends to zero (i.e., $\lim _{t \rightarrow 0} a(t x) \in \operatorname{core}(x, V)$ ).

Proof: Let $a_{1}: R_{++}^{2} \rightarrow R_{++}$be the projection on the first coordinate of $a$.

For every $0<\alpha<1$ the movement of $a_{1}$ along the ray $\left\{x \mid \frac{x^{1}}{x^{1}+x^{2}}=\alpha\right\}$ satisfies that $\frac{d a_{1}}{d T}(\alpha, T)=-\frac{\left.\alpha(1-\alpha) \frac{\partial h}{\partial \alpha}\right|_{\left((\alpha, 1-\alpha), a_{1}\right)}}{\left.\frac{\partial h}{\partial a_{1}}\right|_{\left((\alpha, 1-\alpha), a_{1}\right)}}$ (Equation 5) is continuously differentiable, ${ }^{56}$ and that this derivative is a function only of $a_{1}\left(\alpha e^{T},(1-\alpha) e^{T}\right) .{ }^{57}$

Take $\alpha_{0} \in(0,1)$, and denote $b_{1}(T):=a_{1}\left(\alpha_{0} e^{T},\left(1-\alpha_{0}\right) e^{T}\right)$.

- If for some $T_{0} \in R$ we have $\frac{d b_{1}}{d T}\left(T_{0}\right)=0$, then $b_{1}(t)$ is constant.

We have the differential equation: $\frac{d b_{1}}{d T}(T)=f\left(b_{1}(T)\right)$, for some continuously differentiable function $f$, and

$$
b_{1}(t)=b_{1}\left(T_{0}\right) \quad \forall t
$$

is a possible solution; therefore there exists no other solution. ${ }^{58}$

- If $\frac{d b_{1}}{d T}(t) \neq 0$ for every $t$, then from the continuity of this derivative, it is always positive or always negative; that is, $b_{1}$ is monotonic.

Therefore $b_{1}$ is monotonic.

Thus, $b_{1}(T)$ either has a limit, as $T$ goes to infinity, or is unbounded (keeps ascending to infinity, or descending to minus infinity).

In other words, either:

- $b_{1}(T)$ is not bounded as $T \rightarrow \infty$; hence for some $-\infty<T<\infty$ it is not individually rational. ${ }^{59}$

- $b_{1}(T)$ is bounded as $T \rightarrow \infty$. Therefore $\lim _{T \rightarrow \infty} \frac{d b_{1}}{d T}(T)=0$, and again by Equation 5 and the continuity of the derivatives as a function of $a_{1}$, we get

56 This was already stated earlier, and is a consequence of the assumption of $h$ being $C^{2}$.

57 This means that the "velocity" of the movement of $a_{1}$ depends only on its "location" and not on the "time," and this "velocity" is continuous in the "location."

58 This is a first-order differential equation, and the function $f$ has a bounded derivative in any bounded region (the derivative is continuous, so in any closed region it is bounded).

59 Either $b_{1}(T)$ descends below the individually rational level $r_{1}$, or $b_{1}(T)$ ascends, and causes $h\left(\left(\alpha_{0}, 1-\alpha_{0}\right), b_{1}(T)\right)$ to descend below the individually rational level $r_{2}$ (recall $\left.\frac{\partial h}{\partial a_{1}}<0\right)$. 
$-\frac{\alpha(1-\alpha) \frac{\partial h}{\partial \alpha\left(\alpha(\alpha, 1-\alpha), b_{1}^{*}\right)}}{\frac{\partial a_{1}}{\partial a_{1}}\left((\alpha, 1-\alpha), b_{1}^{*}\right)}=0$, where $b_{1}^{*}=\lim _{T \rightarrow \infty} b_{1}(T)$. That is, $\left.\frac{\partial h}{\partial \alpha}\right|_{\left((\alpha, 1-\alpha), b_{1}^{*}\right)}=0$, which, by Lemma 3 , implies that the limit point belongs to the core (i.e., $\left.\left(b_{1}^{*}, h\left(\left(\alpha_{0}, 1-\alpha_{0}\right), b_{1}^{*}\right)\right) \in \operatorname{core}\left(\left(\alpha_{0}, 1-\alpha_{0}\right), V\right)\right)$.

If $a_{1}(x) \in \pi_{1}(\operatorname{ConvCore}(x, V))$ for any $x$ on this ray, then (by Proposition 9) $a(x)$ is always individually rational. That is:

$$
\lim _{T \rightarrow \infty} a\left(\alpha e^{T},(1-\alpha) e^{T}\right) \in \operatorname{core}((\alpha, 1-\alpha), V) \quad \forall \alpha \in(0,1) .
$$

The same argument applies when $T$ goes to minus infinity. Therefore:

$$
\lim _{T \rightarrow \pm \infty} a\left(\alpha e^{T},(1-\alpha) e^{T}\right) \in \operatorname{core}((\alpha, 1-\alpha), V) \quad \forall \alpha \in(0,1) .
$$

At this point one might think that we have identified a homogeneous consistent value which belongs to the core ${ }^{60}$ but this is not the case because the limit of a consistent value is not necessarily a consistent value. ${ }^{61}$ To be more precise, this limit is indeed a consistent value only if this limit is $C^{1}$, i.e., only if there is a $C^{1}$ selection from the core (by Corollary 10).

\section{Examples}

\subsection{A game with many consistent values}

Consider continuous market games for which the core does not consist of a single point, but of up to three points for every $x \in \Delta_{++}^{2}$.

In the game ${ }^{62}$ with two types of players, and 2 commodities with utility functions:

$$
\begin{aligned}
& u^{1}\left(w_{1}^{1}, w_{2}^{1}\right)=w_{2}^{1}-\frac{1}{2}\left(w_{1}^{1}\right)^{-2}, \\
& u^{2}\left(w_{1}^{2}, w_{2}^{2}\right)=w_{1}^{2}-\frac{1}{2}\left(w_{2}^{2}\right)^{-2},
\end{aligned}
$$

and initial endowment vectors

$$
e^{1}=\left(\frac{2}{7}, 7\right), \quad e^{2}=\left(7, \frac{2}{7}\right)
$$

\section{Example 2.}

The core points for $(\alpha, 1-\alpha) \in \Delta^{2}$ are shown in Figure 5. Let (as in Figure 5) $\alpha^{\prime}$ and $\alpha^{\prime \prime}$ be the two points of boundary between the ranges where core $(\alpha)$ has a unique point, and the range in which it has more than one point.

\footnotetext{
${ }^{60}$ I want to thank the anonymous referee for pointing this out.

${ }^{61}$ As the limit of a $C^{1}$ function may not be differentiable from below (let alone $C^{1}$ ), it cannot fit into any definition of a smooth consistent value.

${ }^{62}$ This is a modification of a game appearing in [7, pp. 521-522 (Example 15.B.2)].
} 


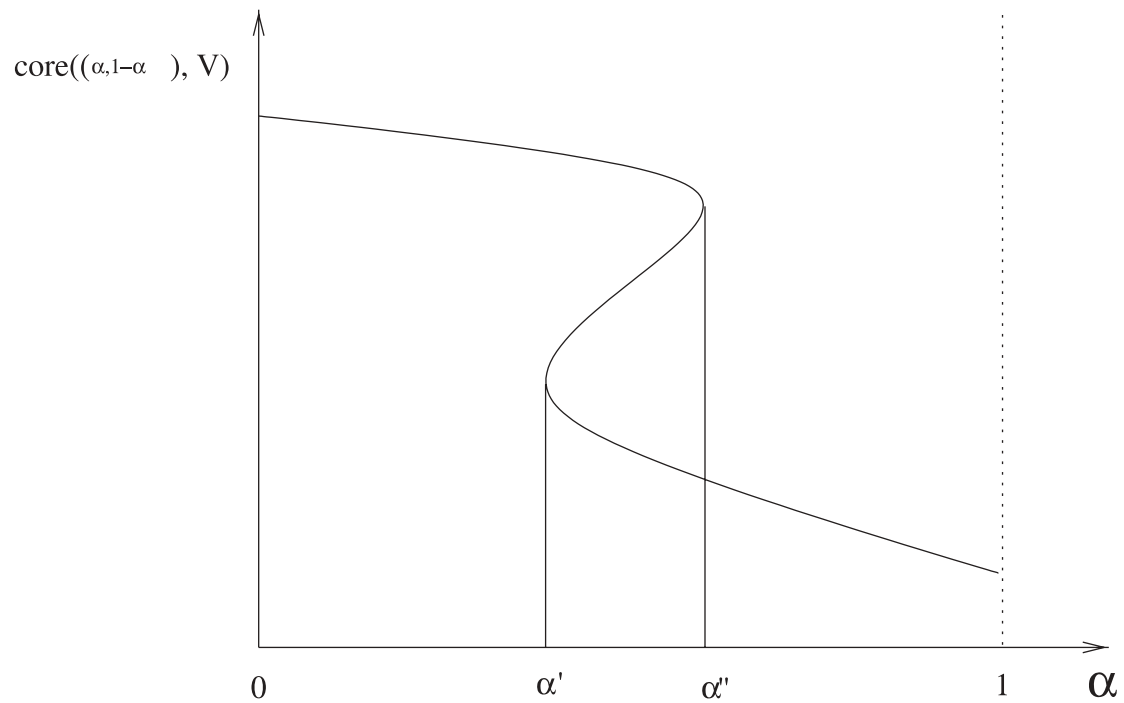

Fig. 5. First coordinate of the points in the core of $((\alpha, 1-\alpha), V)$

\section{Remark:}

1. Since there is no continuous function that always belongs to the core ${ }^{63}$ there exists no consistent value which is smooth and homogeneous of degree 0 (defined on $R_{++}^{2}$ ).

2. There is also no possible selection from the core that is differential from below (for the same reasons as above); therefore there is no semi-smooth consistent value which belongs to the core either.

3. Let $a: R_{++}^{2} \rightarrow R_{++}^{2}$ be a smooth consistent value; then for every $0 \leq \alpha<\alpha^{\prime}$ or $\alpha^{\prime \prime}<\alpha \leq 1$ and any $t>0, a(t \alpha, t(1-\alpha))$ belongs to ConvCore $((t \alpha, t(1-\alpha)), V)=\operatorname{core}((t \alpha, t(1-\alpha)), V)=\operatorname{core}((\alpha, 1-\alpha), V)$.

The derived game function is:

$V(x)=\left\{\begin{array}{l|c}a \in R_{+}^{2} & \begin{array}{c}b=\left(a^{1}-7-\frac{2}{7} \frac{x^{2}}{x^{1}}, a^{2}-7-\frac{2}{7} \frac{x^{1}}{x^{2}}\right) \\ 8 b^{1}\left(\frac{x^{1}}{x^{2}}\right)^{2}+8 b^{2}\left(\frac{x^{2}}{x^{1}}\right)^{2}-3-24 b^{1} b^{2}+16\left(b^{1} b^{2}\right)^{2}=0\end{array}\end{array}\right\}-R_{++}^{2}$,

hence

$$
\begin{gathered}
h\left(x, a^{1}\right)=7+\frac{2}{7} \frac{x^{1}}{x^{2}}+\frac{3 b^{1}-\left(\frac{x^{2}}{x^{1}}\right)^{2}-\frac{x^{1}}{x^{2}}\left(\left(\frac{x^{2}}{x^{1}}\right)^{2}-2 b^{1}\right)^{3 / 2}}{4\left(b^{1}\right)^{2}} \\
\text { for } b^{1}:=a^{1}-7-\frac{2}{7} \frac{x^{2}}{x^{1}}
\end{gathered}
$$

${ }^{63}$ The core is a continuous trajectory, but since pairs of core points "disappear," no continuous function can coincide with the core. 
Let $a: \Delta_{++}^{2} \rightarrow R_{++}^{2}$ be a $C^{1}$ function such that $a(x) \in \operatorname{ConvCore}(x, V)$ for all $x \in \Delta_{++}^{2}$. Define $y: \Delta_{++}^{2} \rightarrow R_{++}$as:

$$
y(x):=\sqrt{\left(\frac{x^{2}}{x^{1}}\right)^{2}-2\left(a^{1}(x)-7-\frac{2}{7} \frac{x^{2}}{x^{1}}\right)} .
$$

If $y$ were defined in this manner on all $R_{++}^{2}$ (from the consistent value $a$ ), then from the consistent value requirements we would get:

$$
t \frac{d y}{d t}=-\frac{2 y^{3}-\left(6 \frac{x^{2}}{x^{1}}+7\right) y^{2}+\left(6\left(\frac{x^{2}}{x^{1}}\right)^{2}+21 \frac{x^{2}}{x^{1}}\right) y-\left(2\left(\frac{x^{2}}{x^{1}}\right)^{3}+14\left(\frac{x^{2}}{x^{1}}\right)^{2}+2 \frac{x^{2}}{x^{1}}\right)}{7 y} .
$$

For $x \in R_{++}^{2}$ such that there are three distinct points in the core $\left\{c_{1}, c_{2}, c_{3}\right\}=\operatorname{core}(x, V)$, and denoting $q_{i}=\left(\left(\frac{x^{2}}{x^{1}}\right)^{2}-2\left(c_{i}^{1}-7-\frac{2}{7} \frac{x^{2}}{x^{1}}\right)\right)^{1 / 2}$,
this equation is equivalent to:

$$
t \frac{d y}{d t}=-\frac{2\left(y-q_{1}\right)\left(y-q_{2}\right)\left(y-q_{3}\right)}{7 y} .
$$

The solution of the above implies that there exist $e_{1}, e_{2}, e_{3}>0\left(e_{1}+e_{2}=e_{3}\right)$ such that the value of

$$
\sqrt[7]{t^{2}} \frac{\left(y(t x)-q_{1}\right)^{e_{1}}\left(y(t x)-q_{3}\right)^{e_{3}}}{\left(y(t x)-q_{2}\right)^{e_{2}}}
$$

is independent of ${ }^{64} t$. This uniquely determines an extension of $y$ to $R_{++}^{2}$, and thereby a consistent value $a: R_{++}^{2} \rightarrow R_{++}^{2}$.

It is easy to $\operatorname{see}^{65}$ that if the original function is $C^{1}$ then the extension is also $C^{1}$; hence it also satisfies the consistent value requirements. See Figure 6 for the behavior of $a_{1}(t x)$ for $x \in \Delta^{2}$ and a few values of $t(t=1, t \ll 1$ and $t \gg 1)$.

The limit of this function as $t$ tends to zero is shown in Figure 7.

And its limit as $t$ tends to infinity is shown in Figure 8.

\subsection{A game with no consistent value}

Here we exhibit a game where no individually rational smooth consistent value exists.

Example 3. Consider the game in the last example (Section 7.1), but with different initial endowment vectors:

\footnotetext{
64 Note that if $q_{1}<y(x)<q_{2}$, then for every $t>0$ there exists a single solution to this equation for $y(t x)$, and this solution holds $q_{1}<y(t x)<q_{2}$. And equivalently for $q_{2}<y(x)<q_{3}$.

65 This is clearly a special case of Section 6.2 , but also quite simple to prove directly.
} 


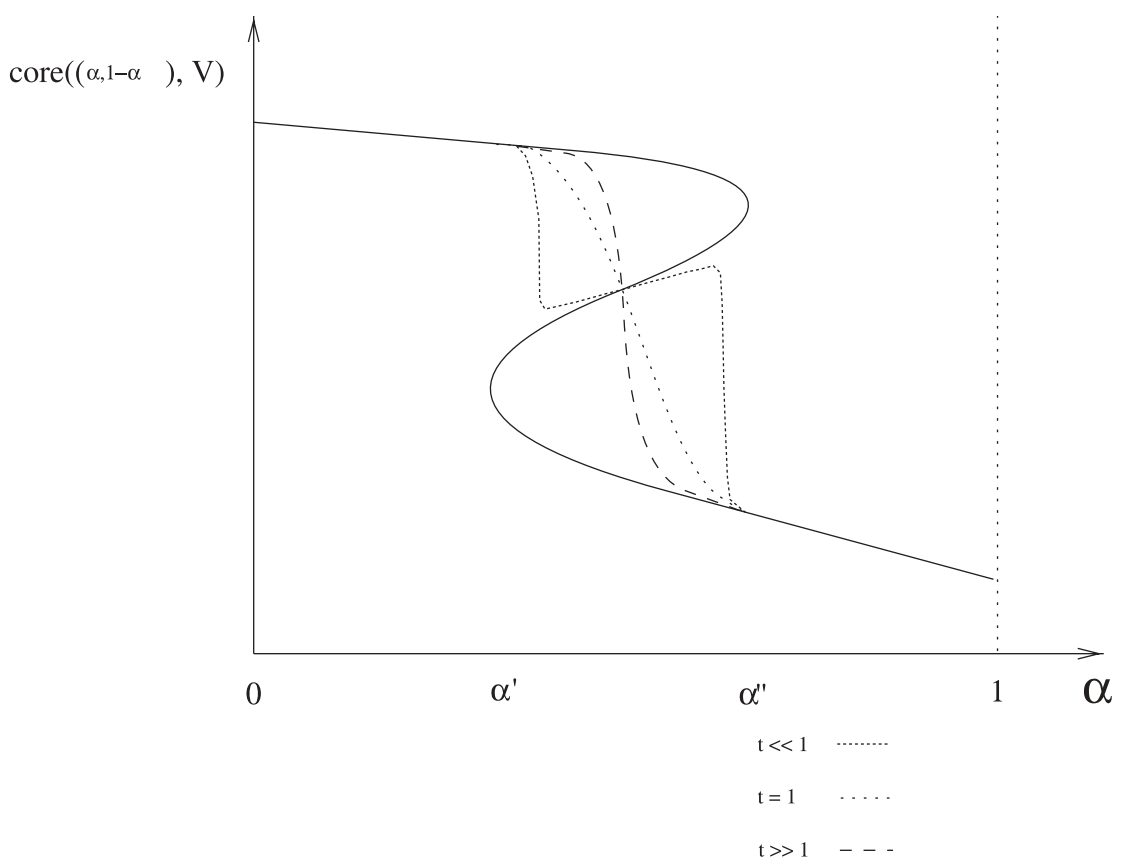

Fig. 6. Flow of the consistent value, for the three core-points example

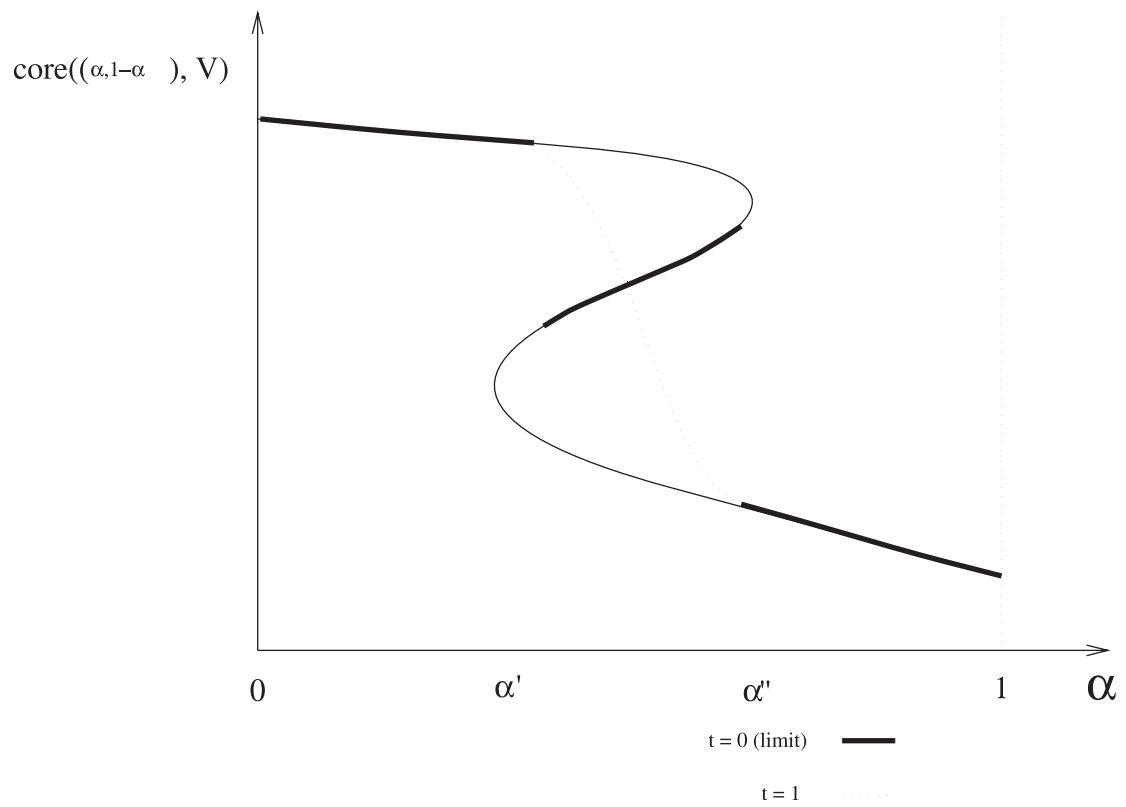

Fig. 7. Limit of the consistent value as $t \rightarrow 0(T \rightarrow-\infty)$ 


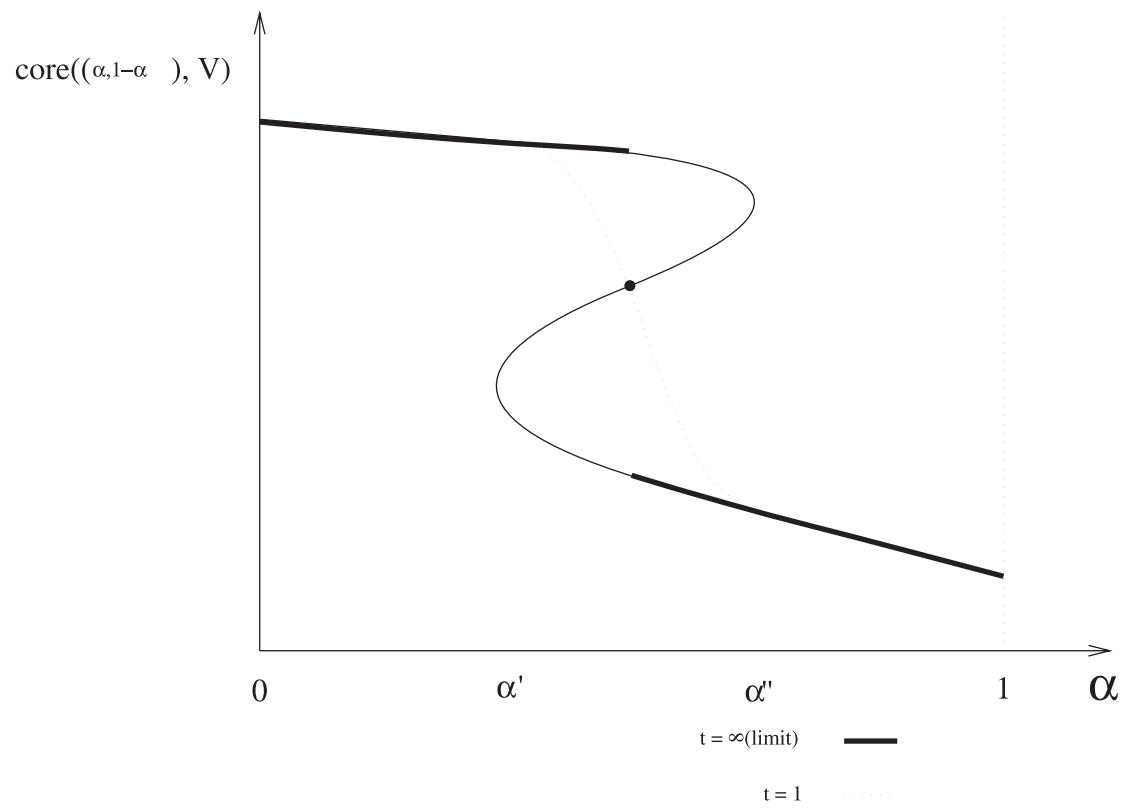

Fig. 8. Limit of the consistent value as $t \rightarrow \infty(T \rightarrow \infty)$

$$
e^{1}=\left(\frac{1}{3}, 5\right), \quad e^{2}=\left(5, \frac{1}{3}\right) .
$$

The core points for $(\alpha, 1-\alpha) \in \Delta_{++}^{2}$ are shown in Figure 9.

In this case, there is a unique core point for every coalition, ${ }^{66}$ but it is not $C^{1}$ as a function ${ }^{67}$ of $x$. Therefore, there is no individually rational smooth consistent value for this game form.

\section{A. Notation}

The following notations are used throughout (for $x, y \in R^{n}$ ):

- $x \cdot y:=\sum_{i=1}^{n}\left(x^{i} y^{i}\right)$.

- $x * y:=\left(x^{i} y^{i}\right)_{i=1, \ldots, n}$.

- $x \leq y$ means: $x^{i} \leq y^{i}$ for every $i$.

- $x<y$ means: $x^{i} \leq y^{i}$ for every $i$, and there exists i such that $x^{i}<y^{i}$.

- $x \ll y$ means: $x^{i}<y^{i}$ for every $i$.

- $\Delta^{n}:=\left\{x \in R_{+}^{n} \mid \sum_{i=1}^{n} x^{i}=1\right\}$.

- $\Delta_{++}^{n}:=\left\{x \in R_{++}^{n} \mid \sum_{i=1}^{n} x^{i}=1\right\}$.

- $\hat{V}(x):=\left\{b \in R^{n} \mid b=x * a\right.$ for some $\left.a \in V(x)\right\}$.

- $\hat{V}_{0}(x):=\hat{V}(x) \cap R_{+}^{n}$.

${ }^{66}$ For coalitions of the type $\left(x^{1}, x^{2}\right)$ such that $x^{1}=x^{2}$ this unique core point is of degree 3 .

${ }^{67}$ The core, as a function, is non-differentiable at $x \in R_{++}^{2}$ such that $x^{1}=x^{2}$ (an unbounded derivative). 


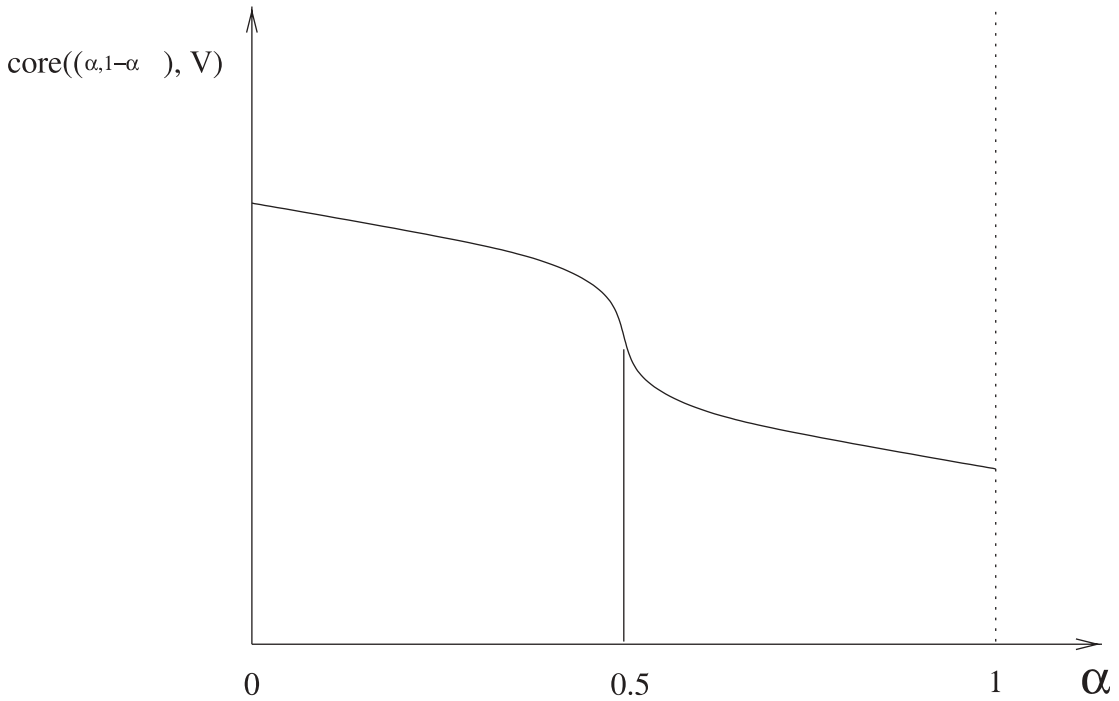

Fig. 9. First coordinate of the points in the core of $((\alpha, 1-\alpha), V)$

- $v(x, p):=\sup \{p \cdot a \mid a \in V(x)\}$.

- $\hat{v}(x, q):=\sup \{q \cdot b \mid b \in \hat{V}(x)\}$.

- $\hat{v}_{0}(x, q):=\sup \left\{q \cdot b \mid b \in \hat{V}_{0}(x)\right\}$.

- $D:=\left\{(x, p) \in R_{++}^{n} \times R_{+}^{n} \mid v(x, p)<\infty\right\}$.

- $\hat{D}:=\left\{(x, q) \in R_{++}^{n} \times R_{+}^{n} \mid \hat{v}(x, q)<\infty\right\}$.

- $\hat{D}^{+}:=\left\{(x, q) \in \hat{D} \mid \hat{v}(x, q)=q \cdot b\right.$ for some $\left.b \in \hat{V}_{0}(x)\right\}$.

\section{References}

[1] Aumann RJ (1975) Values of Markets with a Continuum of Traders. Econometrica 43:611646

[2] Hart S and Mas-Colell A (1996) Bargaining and Value. Econometrica 64:357-380

[3] Hart S and Mas-Colell A (1995) Egalitarian Solutions of Large Games: I. A Continuum of Players. Mathematics of Operations Research 20:959-1002

[4] Hart S and Mas-Colell A (1995) Egalitarian Solutions of Large Games: II. The Asymptotic Approach. Mathematics of Operations Research 20:1003-1022

[5] Hart S and Mas-Colell A (1996) Harsanyi Values of Large Economies: Nonequivalence to Competitive Equilibria. Games and Economic Behavior 13:74-99

[6] Hirsch MW and Smale S (1974) Differential Equations, Dynamical Systems, and Linear Algebra. Academic Press

[7] Mas-Colell A, Whinston MD and Green JR (1995) Microeconomic Theory. Oxford University Press

[8] Maschler M and Owen G (1989) The Consistent Shapley Value for Hyperplane Games. International Journal of Game Theory 18:389-407

[9] Maschler M and Owen G (1992) The Consistent Shapley Value for Games without Side Payments. In Rational Interactions, Selten R, ed, Springer-Verlag, 5-12

[10] Myerson RB (1980) Conference Structures and Fair Allocation Rules. International Journal of Game Theory 9:169-182

[11] Shapley LS and Shubik M (1969) Pure Competition, Coalition Power and Fair Division. International Economic Review 69:337-362 